\title{
Analysis and Evaluation of the Top- $k$ Most Influential Location Selection Query
}

\author{
Jian Chen • Jin Huang • Zeyi Wen • \\ Zhen He • Kerry Taylor • Rui Zhang
}

Received: Aug 22, 2012 / Revised: Nov 08, 2013 / Accepted: Dec 04, 2013

\begin{abstract}
In this paper, we propose a new type of queries to retrieve the top$k$ most influential locations from a candidate set $C$ given sets of customers $M$ and existing facilities $F$. The influence models the popularity of a facility. Such queries have wide applications in decision support systems. A naive solution sequentially scans (SS) all data sets, which is expensive and hence we investigate two branch-and-bound algorithms for the query, namely Estimate Expanding Pruning (EEP) and Bounding Influence Pruning (BIP). Both algorithms follow the best first traverse. On determining the traversal order, while EEP leverages distance metrics between nodes, BIP relies on half plane pruning which avoids the repetitive estimations in EEP. As our experiments shown,

J. Chen

School of Software Engineering, South China University of Technology, Guangzhou, China

J. Huang

Department of Computing and Information Systems, University of Melbourne, Melbourne, Australia

Z. Wen

Department of Computing and Information Systems, University of Melbourne, Melbourne, Australia

Z. He

Department of Computer Science, La Trobe University, Bundoora, Australia

K. Taylor

The Commonwealth Scientific and Industrial Research Organisation (CSIRO), Canberra, Australia

R. Zhang

Department of Computing and Information Systems, University of Melbourne, Melbourne, Australia

E-mail: rui.zhang@unimelb.edu.au
\end{abstract}


BIP is much faster than SS which outperforms EEP, while the worst-case complexity of EEP and BIP is worse than that of SS. To improve the efficiency, we further propose a Nearest Facility Circle Join (NFCJ) algorithm. NFCJ builds an influence R-tree on the influence relationship between customers and existing facilities, and joins the candidate R-tree with the influence R-tree to obtain the results. We compare all algorithms and conclude that NFCJ is the best solution, which outperforms SS, EEP, and BIP by orders of magnitude.

Keywords Reverse Nearest Neighbor; R-tree; Efficiency; Location Selection

\section{Introduction}

A common problem for many business and organizations is to find a suitable place to establish a new facility. For instance, McDonald's may want to introduce a new restaurant into a booming community to compete with other fast food restaurants. A wireless carrier may want to construct a new base station or hotspot for wireless Internet access to a densely populated area to improve its service quality. A scientific organization may want to select a location for a new environmental sensor to capture particular mobile wildlife as the materials for research. A city planner may want to find where to introduce a new public infrastructure such as a drop-in clinic to a flourishing suburb. In most cases, the selection of locations must be made from a given candidate set, e.g., rental commercial properties from a real estate company. This candidate set may be large in some settings, e.g., trulia.com lists more than 43,100 locations for sale in Los Angele, CA, USA [26] and Soufun.com lists more than 339,330 locations for rental in Beijing, China [22]. For business directors, one of the most important indicators used to evaluate a candidate location is the number of customers the newly added facility could attract. In this paper, we investigate the problem of finding the top- $k$ candidate locations that attract the largest number of customers, where $k$ is a user define integer. The top- $k$ locations are of interest because in real applications there are additional factors such as safety and popularity of a brand in a region (for example, fast food is relatively unpopular in suburbs with most European habitants). These may be important factors that will affect the decision but are difficult to quantify. Therefore, top- $k$ results returned by the query can serve as the primary candidates based on which further consideration can be made. In this study, we assume a customer is attracted by his or her nearest facility and the business has the knowledge of customer and existing facility distributions from surveys or past sales records.

An example corresponding to the above problem is shown in Fig. 1, where circles and squares represent customers and facilities, respectively. To distinguish existing facility locations and candidate locations, we use white squares to denote the existing facilities and black squares to denote the candidate facilities. In the figure, the candidate locations are labeled as $c_{1}, c_{2}, c_{3}, c_{4}, c_{5}, c_{6}$. A customer is connected to a candidate location with a dashed line if and only if 


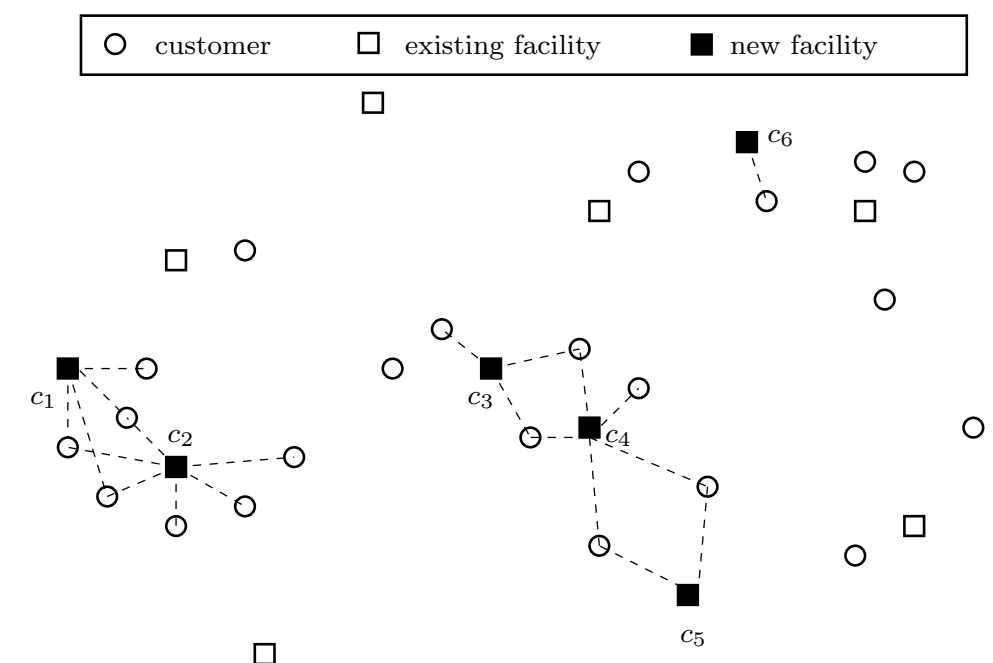

Fig. 1: $c_{1}, c_{2}, c_{3}, c_{4}, c_{5}, c_{6}$ are of influence values $4,6,3,5,2,1$, respectively.

the customer will be influenced by a new facility established at that candidate location. For each candidate location $c_{i}$, the number of customers it attracts can be computed by counting the number of customers connected to it with a dashed line. In this example, the numbers corresponding to $c_{1}, c_{2}, c_{3}, c_{4}, c_{5}, c_{6}$ are $4,6,3,5,2,1$. If a user inputs 3 as the parameter $k$, then the query will return the answer set $c_{2}, c_{4}, c_{1}$.

Please note the number of customers an existing facility location can attract may be reduced by a newly added facility. This follows the idea of competition as McDonald's may want to attract customers from other restaurants such as Burger King. Another example is that a wireless carrier adds a new base station to take load off existing base stations since existing ones are out of capacity or ill-balanced. After adding the new facility, this situation can be improved. In an extreme case, a company may even consider replacing the existing facility with the new facility due to the maintenance cost of keeping the existing facility. In all scenarios, our method can easily address the requirements.

The proposed facility location selection query aims at maximizing the influence of the new facilities, where the influence is defined as the number of customers who perceive the new facility as their nearest facility. Therefore, we formulate the above described problem as the top- $k$ most influential location selection query. We also study the solutions for answering this query in this paper and evaluate their performance with experiments and analysis.

Our main contributions are as follows.

- We formulate a new type of location selection query, namely the top- $k$ most influential location selection query.

- We attempt the popular approach, branch-and-bound to this location selection query and design two algorithms in this paradigm, namely Estimate 
Expanding Pruning (EEP) and Bounding Influence Pruning (BIP) algorithms. Both algorithms follow a best first access order to traverse R-trees and gradually prune the search space. While EEP leverages distance metric between nodes to prune unnecessary computations, BIP relies on half plane pruning, which further avoids repetitive estimation on nodes. According to the experimental results, in terms of CPU time, while BIP outperforms the sequential scan several times, EEP is constantly outperformed by the sequential scan. Additionally, the worst-case complexity of both branchand-bound algorithms is even worse than that of the sequential scan.

- We propose a new algorithm named Nearest Facility Circle Join (NFCJ). It first computes the influence relationship between customers and existing facilities, indexes the relationship in an influence R-tree, and then joins the candidate $\mathrm{R}$-tree with this influence $\mathrm{R}$-tree to obtain results.

- We theoretically analyze all algorithms and conduct an extensive experimental study using data sets with various settings and distributions. The results confirm our complexity analysis and verify that NFCJ is the best solution to the top- $k$ most influential location selection query, which outperforms sequential scan, EEP and BIP by orders of magnitude in terms of both CPU time and the number of I/O operations.

This article is an extension of our earlier poster paper [15]. In the previous poster paper, we introduced the influential location selection query and present the basic ideas of EEP and BIP algorithms. Here in this article, we make the following additional contributions. First, we fully study EEP and BIP with detailed description and analysis. Second, we propose a new algorithm which pre-computes the relationships between customers and existing facilities and indexes them with an R-tree. This R-tree is later joined with the candidate $\mathrm{R}$-tree to return the query result. With analysis of detailed steps, we show that it achieves best $O(n \log n)$ and worst $O\left(n^{2}\right)$ time complexity, beating all other known algorithms for the proposed query. Third, we conduct a comprehensive experimental study to compare all algorithms for the query in terms of both CPU processing time and I/O performance. The results show (i) branch-andbound algorithm BIP is effective in lowering $\mathrm{CPU}$ processing cost when data sets are very large, and (ii) NFCJ significantly outperforms EEP and BIP in terms of both CPU time and the number of I/O operations, which is the best solution for the top- $k$ most influential location selection query.

The remainder of the paper is organized as follows. Section 2 defines the related concepts and top- $k$ most influential location selection query. Section 3 reviews previous studies on related topics. Section 4 [4]describes sequential scan, EEP, BIP, and NFCJ algorithms with the analysis on their complexity. Section 5 presents the experimental results and Section 6 concludes the paper. 
Table 1: Frequently used symbols

\begin{tabular}{l|l}
\hline Symbol & Explanation \\
\hline \hline$C, F, M$ & Sets of candidate, existing facility and customer locations, respectively \\
\hline$c, f, m$ & A candidate location, an existing facility and a customer, respectively \\
\hline$p$ & A point in the data space \\
\hline$t_{C}, t_{F}, t_{M}$ & R-trees on $C, F$ and $M$, respectively \\
\hline$n_{C}, n_{F}, n_{M}$ & A node in $t_{C}, t_{F}$ and $t_{M}$, respectively \\
\hline$r_{C}, r_{F}, r_{M}$ & The MBRs of nodes $n_{C}, n_{F}$, and $n_{M}$, respectively \\
\hline$I_{f}, I_{C}$ & The influence of an existing facility and a candidate facility, respectively \\
\hline$I_{C}^{u}$ & The upper bound of influence for all $c$ indexed by a node $n_{C}$ \\
\hline$I_{C}^{l}$ & The lower bound of influence for all $c$ indexed by a node $n_{C}$ \\
\hline$I_{\delta}$ & The $k^{t h}$ greatest influence value of candidates seen so far \\
\hline$L_{C}, L_{F}, L_{M}$ & The priority list of nodes $n_{C}, n_{F}$, and $n_{M}$, respectively \\
\hline$n_{C} \cdot S_{M}, n_{F} \cdot S_{M}$ & The unpruned customers of nodes $n_{C}$ and $n_{F}$, respectively \\
\hline$n_{M} \cdot S_{C}, n_{M} \cdot S_{F}$ & The unpruned candidates and existing facilities of node $n_{M}$, respectively \\
\hline$n_{C} \cdot R$ & The influence region of a node $n_{C}$ \\
\hline$n_{C} \cdot S_{F}$ & The relevant existing facilities set of a node $n_{C}$ \\
\hline$n_{C} \cdot S_{F}^{+}$ & The outer relevant existing facilities set of a node $n_{C}$ \\
\hline$n_{C} \cdot S_{F}^{-}$ & The inner relevant existing facilities set of a node $n_{C}$ \\
\hline &
\end{tabular}

\section{Preliminary Concepts and Problem Formulation}

In this section, we first introduce the related concepts and definition of location influence, based on which we then propose a novel query to select the optimal location for a new facility.

\subsection{Location Influence}

We present formal definition of reverse nearest neighbor and location influence first. Table 1 lists frequently used symbols in the paper.

Definition 1 (Reverse Nearest Neighbors) The customers who perceive a facility as their nearest facility are reverse nearest neighbors of this facility. Let $d(f, m)$ denote the Euclidean distance between $f$ and $m, \min (m, F)$ denote the minimum distance between $m$ and any $f \in F, f . R N N(F, M)$ denote the reverse nearest neighbors of $f \in F$, then $f \cdot R N N(F, M)=\{m \in M \mid d(m, f)=$ $\min (m, F)\}$.

Note this definition presents the bichromatic version of the reverse nearest neighbors problem, where objects are divided into two categories. The reverse nearest neighbors of an object in such scenario always come from the opposite category [16]. 


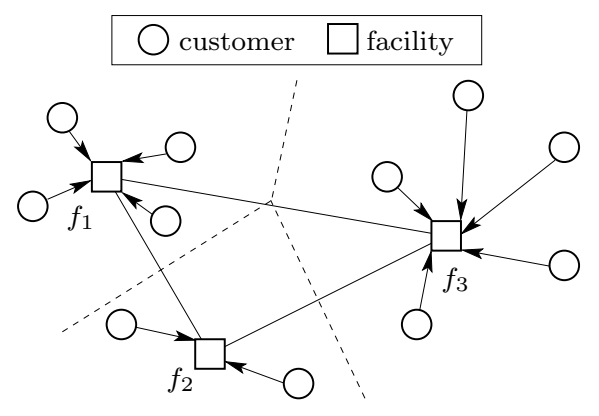

Fig. 2: $I_{f 1}=4 ; I_{f 2}=2, I_{f 3}=5$

When evaluating the popularity of a facility, counting the number of its reverse nearest neighbors would be a sensible indicator because in many scenarios such as marketing and city planning, specific individuals are of less interest than the overall number of customers. We have:

Definition 2 (Location Influence) The influence of a location is the number of its reverse nearest neighbors. Let $I_{f}$ be the influence of facility $f$, then $I_{f}(F, M)=|f \cdot R N N(F, M)|$.

Fig. 2 demonstrates an example of facility influence, where circles denote customers and squares denote facilities. In the figure, the dash lines are perpendicular bisectors between each pair of facilities. Easily, $I_{f 1}=4, I_{f 2}=2$, $I_{f 3}=5$.

Here we assume each customer location contributes the same 1 unit influence. Yet, our problem setting can be generalized to take variable units into consideration. For the sake of brevity, we follow the 1 unit setting for the rest of this paper.

Instead of focusing on the specific reverse nearest neighbors, Definition 2 can be used as the criteria for ranking facilities based on their attractiveness for various applications. The influence based location problem has wide applications in fields like commercial marketing and community planning. Selecting an optimum location is of great interest when different locations offer diverse potential profits. In addition, the influence location selection problem generally faces massive data sets in the real world where there can be numerous facilities and customers. This calls for an efficient solution to make the query viable for integration into decision making systems.

\subsection{Influence Maximization for A New Facility from Candidate Set}

When selecting the location for a new facility, we aim to find the one with highest potential to be popular among all existing facilities. Using location influence as an indicator, it is possible to select the optimum location for a to-be built facility. Specifically, we can evaluate the potential popularity of a location by adding a new facility on it, and then computing the influence of that new facility. The influence of the new facility turns out to be an indicator 


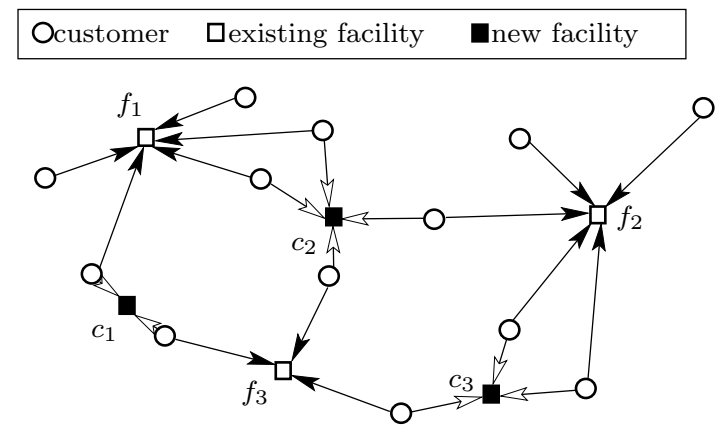

Fig. 3: $C=\left\{c_{1}, c_{2}, c_{3}\right\} ; I_{c 1}=2, I_{c 2}=4, I_{c 3}=3$; Top-2 influential candidates are $c_{2}, c_{3}$

of desirability of the location. Obviously, the more customers the new facility can influence, the better the location. This leads to the following definitions:

Definition 3 (Potential Influence) Let $C$ denote a candidate set of available locations for a to-be built facility. The potential influence $I_{c}^{\prime}$ of a candidate location $c \in C$ is the influence it earns when it is added into the existing facility location set, i.e. $I_{c}^{\prime}=I_{c}(F \bigcup\{c\}, M)$.

In the remainder of the paper, when there is no ambiguity, we use $I_{f}$ to denote $I_{f}(F, M)$ and $I_{c}$ to denote $I_{c}(F \bigcup\{c\}, M)$ for brevity.

Definition 4 (Top- $k$ Most Influential Locations) Given a set $C$ of candidate locations for the new facility, the top- $k$ most influential locations are $k$ locations in $C$ with the largest potential influence.

If multiple candidate locations have the same potential influence value, there might be ties when selecting the top- $k$ candidates. To resolve this, we arbitrarily choose some of them as the answer. For example, when more than $k$ candidates are of same largest potential value, we simply pick $k$ of them as the answer.

Fig. 3 gives an example for this potential influence. In the figure, the circles denote customers, the white squares denote existing facilities, black squares denote potential new facilities. As illustrated, if $c_{1}$ is added into existing facilities, it can influence two customers from $f_{1}$ and $f_{3}$ (customers with white arrows pointing to $c_{1}$ ); if $c_{2}$ is added, it can influence four customers from $f_{1}, f_{2}$, and $f_{3}$; if $c_{3}$ is added, it can influence three customers from $f_{2}$ and $f_{3}$ . Therefore, the potential influence of $c_{1}, c_{2}$, and $c_{3}$ are $2,4,3$, respectively. According to definition 4, the top-2 most influential locations among $C$ are $c_{2}$ and $c_{3}$.

\section{Related Work}

\subsection{Reverse Nearest Neighbor}

Korn and Muthukrishnan [16] first proposed the reverse nearest neighbor $(\mathrm{RNN})$ query and define the RNNs of an object $o$ to be the objects whose 
respective nearest neighbor is $o$. In the same paper [16], Korn and Muthukrishnan propose an RNN-tree based solution to the RNN query, where the RNN-tree is an R-tree [14] variant that indexes nearest neighbor (NN) circles of the data objects rather than the data objects themselves. Here, the NN circle of an object is defined to be a circle that centers at $o$ with its radius being the distance between $o$ and $o$ 's nearest neighbor. Based on the NN circles, to find the RNN of an object $o$ only requires checking which objects' NN circles enclose $o$. Applying this idea to our top- $k$ most influential query gives the Nearest Facility Circle (NFC) algorithm. However, the RNN-tree based solution has two major drawbacks. One is that it requires the extra maintenance of an RNN-tree. The other is that it requires precomputing the NN circles. Therefore, this solution can not handle objects with frequent updates. To solve the first problem, Yang and Lin [33] propose to integrate the NN circle information into an R-tree, so that the resultant R-tree can be used to process RNN queries as well as other common types of queries, thus avoiding the maintenance of an extra RNN-tree. To solve the second problem, Stanoi et al. [23] propose an approximation-refinement framework to compute the RNNs on the fly, so that no precomputation is needed. While these methods work well for processing a single RNN query, they are not designed to compute RNNs for a large number of objects at the same time, which is one of the key difficulties in many facility location problems. Thus, the RNN problem can be viewed as a sub problem of the facility location problems. Recent progress on improving the efficiency of answering $\mathrm{R} k \mathrm{NN}$ query can be found in [25], [30], and [1]. Techniques proposed for similarity (nearest neighbor) search such as bulk loading index construction [3] and pre-computing key function values for similarity search [9] can also be helpful in RNN search.

\subsection{Location Distance Minimization}

Min-dist facility location problems aim to minimize the average distance between customers and their respective nearest facilities. Zhang et al. [34] propose to find an optimal location $c$ in a given region $Q$ such that if a new facility is built on $c$, the average distance between the customers and their respective nearest facilities is minimized. Mouratidis et al. [17] study the $k$ medoid query and the $k$ median query, which aim at finding a set $C^{\prime}\left(C^{\prime} \subset C\right)$ of $k$ locations from a set $C$ to minimize the average distance between locations in $C^{\prime}$ and locations in $C$. Similar to our problem settings, given client set and existing facility set, Qi et al. [19] propose a new min-dist location selection query which aims to pick the best location from a candidate set to minimize the average distance between a client and its nearest facility. Besides also introducing the NFC algorithm to solve the problem, they propose a novel method to answer the query without the need to construct a spatial index in advance. The performance of this method is verified to be close to the best algorithm via extensive experiments. All these studies are distance based optimization problems and are different from our influence based optimization problem because they focus 


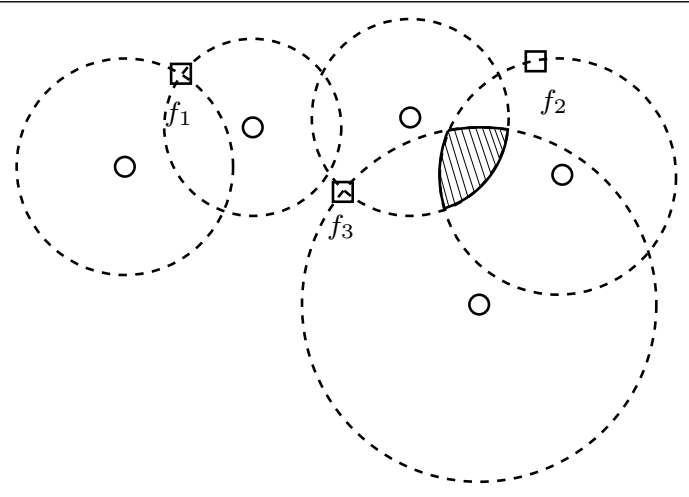

Fig. 4: Example of the MAXCOV problem

on optimizing the overall performance of all the facilities while our problem focuses on optimizing the performance of one particular facility. Hence, their solutions are not applicable.

\subsection{Location Influence Maximization}

Max-inf facility location problems aim at maximizing the influence values of the locations, where the influence of a location $c$ is defined by the number of customers $c$ attracts. Cabello et al. [5] propose a facility location problem based on the MAXCOV optimization criterion, which is to find regions in the data space that maximize the numbers of RNNs for the points in these regions. Fig. 4 gives an example, where the gray region is the optimal region. Points in this region have three RNNs, while any point outside of this region has at most two RNNs. They introduce the concept of nearest location circle (NFC) to solve the problem, where the NFC of a customer $m$ is a circle centered at $m$ with its radius being the distance between $m$ and $m$ 's existing nearest facility. To find the solution for the MAXCOV criterion based problem is to find the regions that are enclosed by the largest number of NFCs, which requires complex computations. The study give a theoretical analysis, but no efficient algorithm is presented.

Xia et al. [31] propose the top- $t$ most influential sites problem and a branchand-bound algorithm to solve it. This problem finds the top- $t$ most influential existing sites within a given region $Q$. It does not consider any candidate locations for a new facility. That is to say, in their work, the influence computation is based on all existing facilities, and the influence comparison is between all existing facilities. In our work, the influence computation is based on the set obtained by adding each candidate location into the existing facility set, and the influence comparison is between all candidate locations. The only possible way to reuse their solution is to first add each candidate into the existing set to compute the top- $t$ influential locations in this new set and then rank all candidate locations by their influences to return the top- $k$ locations. Yet because the added candidate location is not necessarily among the top- $t$ 
answer of its corresponding new set, the adapted solution cannot guarantee the correct answer unless we set $t$ to the size of the new set, i.e., $|F|+1$. Hence, there is no straightforward way to adapt their solution to solve our problem.

One highly related problem is also studied in [29]. Yet the expected answer for their most influential location query is a region where the new facility being added could earn the same maximum influence values. To achieve an efficient solution, they take advantage of region-to-point transformation to tighten the search space dramatically. The proposed method is further extended in [28] to handle similar problem in any $\ell_{p}-$ norm space of two and three dimensionality. However, their problem selects the optimal region while our problem selects top- $k$ influential locations from a candidate set. There may be no candidate in the optimal region returned by their solutions. Therefore, their solutions do not apply to our problem.

Another similar study is [32], where assumption that customers will only visit their nearest facilities is relaxed such that all facilities within the distance of $(1+\alpha) d$ from the customer might be visited, where $d$ is the distance between a customer and her nearest neighbor and $\alpha$ is a user input parameter indicating how much further a user would like to travel for a non-nearest facility. Furthermore, the study also gives a greedy solution for finding the $k$ locations for adding $k$ new facilities simultaneously to gain the overall maximum influence. A grid-based technique is used to divide the space and return the grid with highest potential influence as the answer. Again, since the problem setting does not consider the candidate set, applying their method to our problem will not give direct solutions since we would need to return multiple grids until $k$ candidates are found enclosed in these returned grids.

Du et al. [11] propose to find a point from a continuous candidate region that can maximize the influence value. They use $\ell_{1}$ distance and have a strong assumption that all the roads are either horizontal or vertical. We consider $\ell_{2}$ distance, which is a more general problem setting. More importantly, we consider a candidate location set instead of a candidate region. This is a more practical problem setting because in many real applications, we can only choose from some candidate locations (e.g. a McDonald's restaurant can only be opened at a place for lease or sale, rather than anywhere in a region). Cheema et al. [7] propose to find an influence zone for a query location $c$, where the customers inside this zone form exactly the reverse $k$ nearest neighbor $(R k N N)$ query result of $c$. Here, a $\mathrm{R} k \mathrm{NN}$ query retrieves all the data points that have $c$ as one of their $k$ nearest neighbors. They use a method to compute Voronoi cells on the fly for the query location to obtain its R $k$ NNs. The proposed method is further rigorously analyzed in [8], and shown to be available when the dimensionality is more than two and there is data update. Compared to this problem, our problem focuses on the number of RNNs of the candidate locations instead of specific locations of the RNNs. Recently, a similar influential location selection problem with capacity limit is studied [24]. The major difference between this study and the study presented in this paper is twofolds: first, the capacity of facility is out of consideration in the study here, therefore the optimization goal is for the new facility only 
Table 2: Existing studies on location influence maximization problem and our problem

\begin{tabular}{c|c|c|c|c|c}
\hline Study & Input & Output & Influence Definition & Space & Solution \\
\hline \hline$[5]$ & $M$ & Regions & Number of RNNs & $\ell_{2}$ & NFC \\
\hline$[31]$ & $M, F$ & Top- $k \in F$ & Number of RNNs & $\ell_{2}$ & Branch-and-bound \\
\hline$[29,28]$ & $M, F$ & $\begin{array}{c}\text { Regions for } \\
\text { a new facility }\end{array}$ & Number of RNNs & $\ell_{p}$ & $\begin{array}{c}\text { Region-to-point } \\
\text { transformation }\end{array}$ \\
\hline$[32]$ & $M, F$ & $\begin{array}{c}\text { Regions for } \\
\text { a new facility }\end{array}$ & $\begin{array}{c}\text { Number of } m \in M \text { within } \\
\text { distance of their }(1+\alpha) \text { NN }\end{array}$ & $\ell_{2}$ & $\begin{array}{c}\text { Greedy and grid } \\
\text { partitioning }\end{array}$ \\
\hline$[7,8]$ & $M, F$ & Index structure & Number of R $k N N$ & $\ell_{2}$ & Voronoi cell \\
\hline$[24]$ & $M, F$ & $\begin{array}{c}\text { Regions for } \\
\text { new facilities }\end{array}$ & $\begin{array}{c}\text { Number of RNN } \\
\text { constrained by capacity }\end{array}$ & $\ell_{2}$ & $\begin{array}{c}\text { NFC and } \\
\text { heuristic pruning }\end{array}$ \\
\hline$[13]$ & $M, F$ & $\begin{array}{c}\text { Network segments } \\
\text { for new facilities }\end{array}$ & Number of RNNs & $\begin{array}{c}\text { Spatial } \\
\text { network }\end{array}$ & Branch-and-bound \\
\hline$[21]$ & $M, F$ & Top- $k f \in F$ & Number of RNNs & $\begin{array}{c}\text { Path } \\
\text { trajectory }\end{array}$ & Branch-and-bound \\
\hline$[36]$ & probabilistic $M, F$ & Top- $k f \in F$ & Expectation of RNNs & $\ell_{2}$ & Branch-and-bound \\
\hline$[11]$ & $M, F$, & Top $c \in C$ & Number of RNNs & $\ell_{1}$ & Branch-and-bound \\
\hline$[12]$ & $M, Q, d$ & Top $f \notin Q$ & Number of $m \in M$ & $\ell_{2}$ & Branch-and-bound \\
\hline This paper & $C, F, M$ & Top- $k c \in C$ & Number of RNNs & $\ell_{2}$ & Branch-and-bound, NFCJ \\
\hline
\end{tabular}

instead of for all facilities to achieve maximum influence; only facilities in the candidate set is considered here, while the study in [24] attempts to find all locations in the data space that would achieve the optimization. This difference makes the solution there inapplicable to our problem.

While most studies deal with problems in either Euclidean space or $\ell_{p}-$ norm space, the location influence maximization problem is also studied in spatial networks. Ghaemi et al. [13] tackle the problem where both query objects and sites reside on the spatial network. Shang et al. [21] propose to represent the facility locations by path trajectories. This way, the most accessible locations could be selected based on the number of path trajectories that perceive the location as their nearest facilities. When data sets contain uncertain instances, Zheng et al. [36] formulate the most influential locations as those with highest expected ranks. To efficiently answer the query, the authors propose several pruning rules and a divide-and-conquer paradigm to eliminate search space in terms of locations to be computed and the number of possible worlds needed to be checked. Clearly, none of these studies consider an additional candidate set for the to-be added facility, making their solutions inapplicable to our problem.

Unlike the above problems, which define the influence values based on the cardinalities of RNN sets, Gao et al. [12] propose to find the optimal location $f$ outside a given region $Q$ based on the number of customers in $Q$ whose 
distances to $f$ is within a given threshold $d$. We consider neither the specific region $Q$ nor the given threshold $d$ in our study, which makes their solution inapplicable to our problem.

To summarize, Table 2 lists existing studies and our study presented in this paper based on their inputs, outputs, assumptions on formulation the problem and the proposed solutions.

\section{Solutions}

In this section, we comprehensively study four solutions for top- $k$ most influential location selection query. To begin with, we follow the definition of the problem and present a rather naive solution, which is based on performing a sequential scan (SS) on all data sets. Although this solution returns correct answer, its efficiency is unsatisfactory since it accesses the data sets intensively and performs repetitive computations. We further investigate two R-tree based branch-and-bound solutions. The R-tree is a widely used index structure designed specifically for spatial data[14]. Each spatial object is associated with a Minimum Bounding Rectangle (MBR). Multiple MBRs are then grouped as nodes in upper levels of the tree, which are again associated with bigger MBRs bounding all MBRs in the corresponding group. To perform queries on an Rtree, typically we traverse the tree using the MBR corresponding to a node as the indicator to decide whether its children of that node should be accessed. Both branch-and-bound methods index all three data sets with R-trees or its variant, and rely on estimating the influence bounds for candidate locations to tighten the search space. One of them, named Estimation Expanding Pruning (EEP), uses distance metrics between MBRs to gradually refine the estimation during traversing all three trees. The other algorithm, named Bounding Influence Pruning (BIP), leverages Voronoi-cell based half plane pruning properties reduce the search space. Both EEP and BIP are of complexity $O(n \log n)$ in the best case, which is better than the $O\left(n^{2}\right)$ complexity of SS. Yet, the worst case complexities of EEP and BIP are $O\left(n^{4}\right)$ and $O\left(n^{3}\right)$, respectively, which are far from competitive. To overcome this, we proposed a new algorithm named Nearest Facility Circle Join (NFCJ). This solution indexes the nearest facility circle instead of the location for each customer and transforms the most influential location selection query into join query between the influence tree and the candidate tree. NFCJ algorithm achieves $O(n \log n)$ in the best case and $O\left(n^{2}\right)$ in the worst case. We concluded that NFCJ is the best solution for the top- $k$ most influential location selection query based on the analysis on complexity of all algorithms.

\subsection{Sequential Scan}

As defined in Definition 4, the problem can be solved in a straightforward manner following the definition. To select the $k$ most influential locations in 
the candidate set $C$, we first compute the exact influence of each candidate $c \in C$ then simply return the $k$ largest. The naive implementation of this idea is to use sequential scan to obtain the candidate influence. For each $c$, we obtain its influence value by adding it to the existing facility set $F$ and scanning the customer set $M$ to find reverse nearest neighbors, namely $m$ s which have $c$ as their nearest facility. This requires $|C||F||M|$ number of scans.

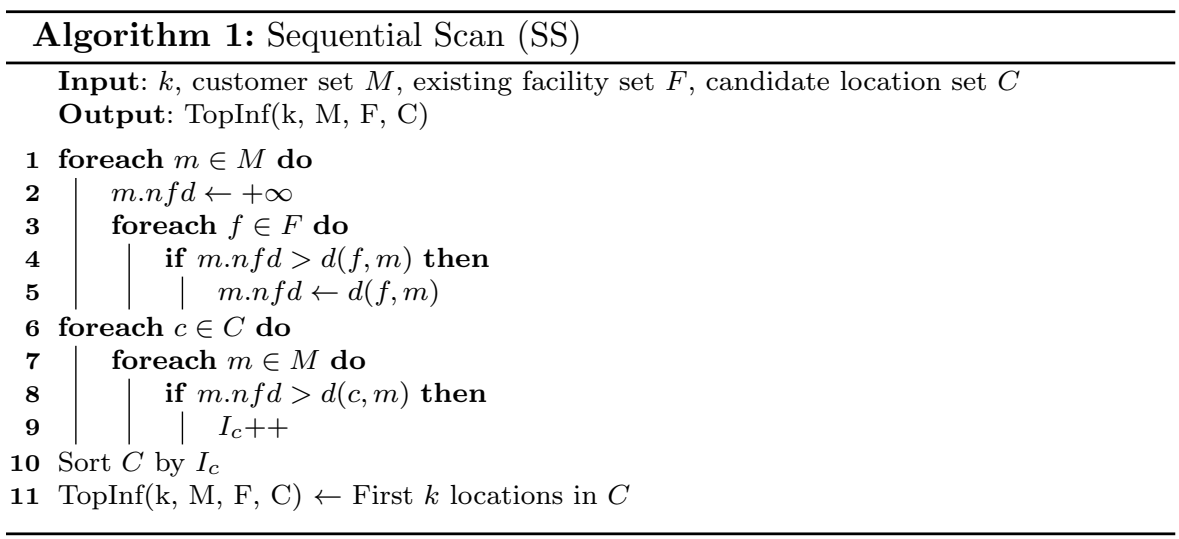

Notice that the set $M$ is repetitively scanned for existing facilities $f \in F$ when computing influence for each $c$. These repetitive scans can be avoided by first scanning the customer set $M$ and the existing facility $F$, then storing the distance between each $c$ and its nearest facility for further use. Let this distance be called $n$ earest $f$ acility distance $(n f d)$. When computing the influence for candidate $c$, we can scan $M$ again to find which $m \in M$ perceives $c$ as the nearest facility by simply checking whether the distance between $c$ and $m$ is within the $n f d$ corresponding to $m$. Due to the fact this method only needs sequential scans on data sets, we name it the Sequential Scan algorithm.

The algorithm can be summarized as Algorithm 1, where m.nfd is the nearest facility distance of $m$ and $d(a, b)$ is the distance between location $a$ and location $b$. As shown in the pseudo-code, it is easy to find that the sequential scan requires $|F||M|+|C||M|$ distance computations, leading to a time complexity of $O\left(n^{2}\right)$.

The sequential scan is far from efficient of solving top- $k$ most influential location problems due to the fact it relies on intensive scans on all of three data sets and computes unnecessary influence for weakly influential candidates, which are of less interest in the problem. Also, repetitive scans on set $M$ is undesirable since in reality, this set tends to be the largest among all sets. In following sections, we come up with two algorithms relying on heuristics to prune search space and improve efficiency in terms of CPU time. 


\subsection{Estimation Expanding Pruning}

To prepare the pruning of the search space, we index sets $C, F$ with R-trees and $M$ with an aggregate $\mathrm{R}$-tree for more efficient access and heuristic operations. Let $t_{C}, t_{F}, t_{M}$ denote these R-trees. Recalling that our problem asks for top- $k$ most influential locations, it is desirable to estimate the influence for candidates before computing their exact influence and to use this information to eliminate unpromising candidates at an early stage. This way, we can avoid exhaustive computations by taking advantage of influence value distribution among candidates.

In this section, we propose a distance based technique to help us estimate the influence of candidate locations. The distances between customers and their nearest existing facilities as well as the possible number of customers that could be influenced by the candidate facility are estimated. With these estimations, the solution traverses $t_{C}$ in a best first order determined by $\mathrm{im}$ portance of that node, which depends on both estimated influences and the number of candidates the node's Minimum Bounding Rectangle (MBR) encloses, to quickly find the top- $k$ candidates.

When an internal node of $t_{C}$ is accessed, each of its child nodes is evaluated for its importance. Since this operation naturally expands the search space, we call it the expanding operation on an index tree. $t_{F}$ and $t_{M}$ are also expanded so that the estimations on distances and influences can be gradually refined, which brings more effective pruning in return. As estimation and expanding operations play the major role in the solution, it is named the Estimation Expanding Pruning (EEP) algorithm.

Trees $t_{C}, t_{F}, t_{M}$ are traversed in a best first order by maintaining priority lists $L_{C}, L_{F}$, and $L_{M}$, which contain entries corresponding to the to-be accessed nodes. These entries also record the importance values for the nodes and some influence relation information presented by related entries in other lists. Initially, only the roots of trees are stored in the corresponding lists. As the traversal proceeds, lists are accessed in $L_{M}, L_{F}, L_{C}$ order, most important entries in lists are expanded and entries corresponding to their child nodes are re-inserted into the lists. The repetitive accesses terminate when the top- $k$ candidates have been found. Specifically, we maintain a sorted list for all computed influence values in descending order. Once all upper bound $I_{C}^{u}$ of influences for nodes in the tree are smaller than the $k^{\text {th }}$ influence value in that list, no more candidate location remaining in the tree could serve as the query answer since none of them could have an influenced value greater than the $k^{\text {th }}$ computed one. Hence, the algorithm could terminate at an early stage. Algorithm 2 shows the high level algorithm of EEP.

In lines 4 to 6 of Algorithm 2, the entries with greatest importance value are selected, the corresponding nodes are expanded, and the importance values and influence relation information of their child nodes are computed so that promising nodes are re-inserted into the lists as entries while unpromising nodes are discarded directly. Details for this procedure is given in Section 4.2.3. Before taking a more detailed look, we will first give the definitions on 


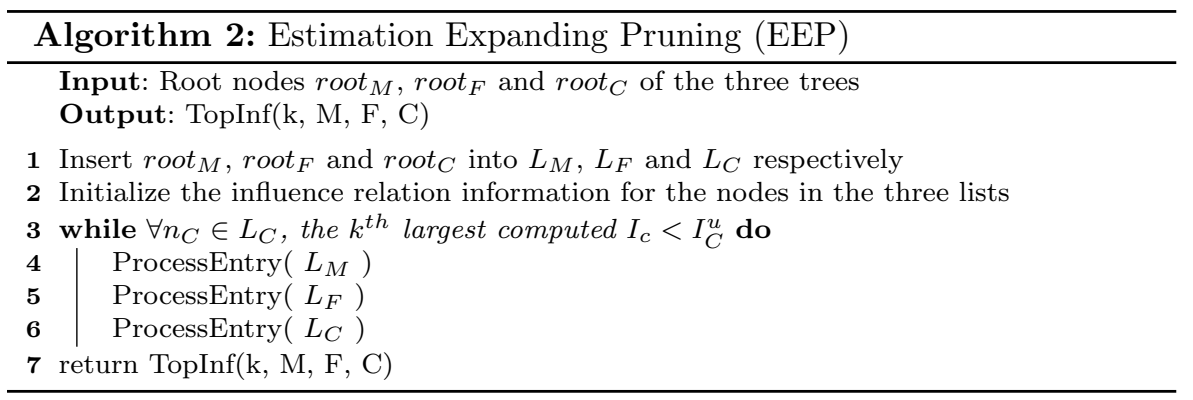

hints used in the procedure, which are stored in the entry with corresponding node. Specifically, influence relation information is introduced in Section 4.2.1 and importance values of nodes are defined in Section 4.2.2. The run-time complexity of EEP is given in Section 4.2.4.

\subsubsection{Influence relation information of nodes}

The influence relation information of a node $n \in t$ contains sets of nodes from trees other than $t$ which either potentially influence $n$ if $n$ represents customers or potentially influenced by $n$ if $n$ represents facilities. These sets are named influence relation sets. In addition, some distances between node $n$ and nodes in influence relation sets are also stored for efficient access and computation. This information together helps in efficiently determining the importance of a node, as described in Section 4.2.2, as well as pruning the search space when processing entries, as elaborated in Section 4.2.3.

Specifically, for $n_{C}$, in order to estimate the influence for candidates enclosed in its $r_{C}$, a set named $n_{C} . S_{M}$ is maintained as the set of nodes $n_{M}$ which might be influenced by any $c$ enclosed by $r_{C}$. For $n_{F}$, similar to that for $n_{C}$, a set $n_{F} . S_{M}$ is maintained for estimating the influence of facility location represented by $n_{F}$. For $n_{M}$, two sets $n_{M} . S_{F}$ and $n_{M} . S_{C}$ are maintained. Set $n_{M} . S_{F}$ contains $n_{F}$ such that facility $f$ enclosed by $r_{F}$ may influence any customer $m$ enclosed by $r_{M}$. Similarly, set $n_{M} . S_{C}$ contains nodes $n_{C}$ such that candidate facility $c$ enclosed by $r_{C}$ may influence any customer $m$ enclosed by $r_{M}$. The method to determine these sets are elaborated in the later part of this section. Fig. 5 shows an example, where $L_{C}=\left\{n_{C 1}, n_{C 2}, n_{C 3}\right\}$, $L_{F}=\left\{n_{F 1}, n_{F 2}, n_{F 3}\right\}, L_{M}=\left\{n_{M 1}, n_{M 2}, n_{M 3}\right\}$. As demonstrated in the figure, $n_{C 1} . S_{M}=\left\{n_{M 1}, n_{M 2}\right\}, n_{C 2} . S_{M}=\left\{n_{M 1}\right\}, n_{C 3} . S_{M}=\left\{n_{M 3}\right\} ; n_{F 1} \cdot S_{M}=$ $\left\{n_{M 1}, n_{M 2}\right\}, n_{F 2} . S_{M}=\left\{n_{M 3}\right\}, n_{F 3} . S_{M}=\left\{n_{M 3}\right\} ; n_{M 1} . S_{C}=\left\{n_{C 1}, n_{C 2}\right\}$, $n_{M 1} . S_{F}=\left\{n_{F 1}\right\}, n_{M 2} . S_{C}=\left\{n_{C 1}\right\}, n_{M 2} . S_{F}=\left\{n_{F 1}\right\}, n_{M 3} . S_{C}=\left\{n_{C 3}\right\}$, $n_{M 3} \cdot S_{F}=\left\{n_{F 2}, n_{F 3}\right\}$.

In order to compute the influence relation sets for a given node efficiently, we introduce three distance metrics between nodes. Given two nodes, which are represented by their MBRs $r_{1}$ and $r_{2}$, the $\operatorname{MinDist}\left(r_{1}, r_{2}\right)$, and the $\operatorname{MaxDist}\left(r_{1}, r_{2}\right)$ are respectively the minimum distance and the maximum distance between 


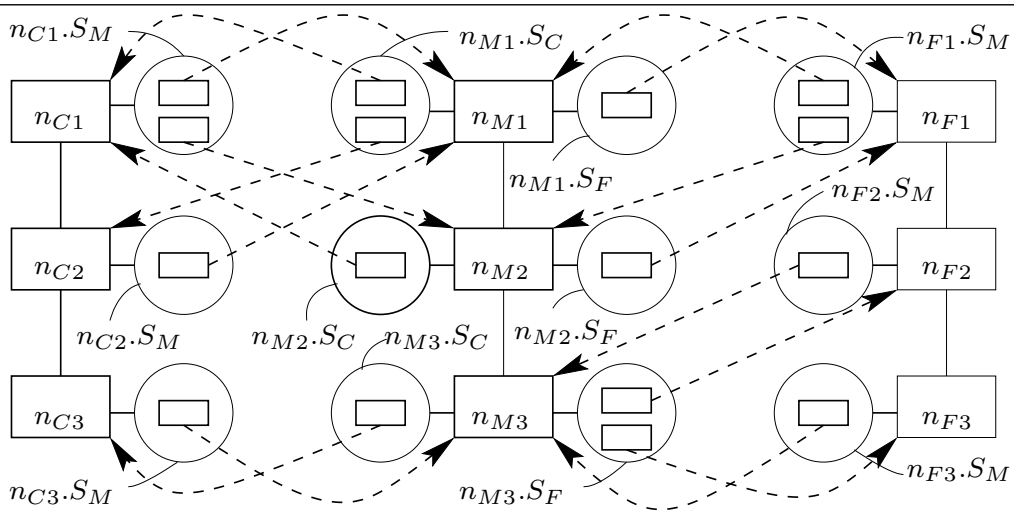

Fig. 5: An example for influence relation sets on list $L_{C}, L_{F}, L_{M}$

any pair of points, one enclosed by $r_{1}$ and the other in $r_{2}$. The distance metric MinExistDist ${ }_{r_{2}}\left(r_{1}\right)$, which is introduced in [31], is defined as the minimum upper bound of the distance for a point in $r_{1}$ to its nearest point in $r_{2}$. In other words, it is possible to find the nearest point in $r_{2}$ for any point in $r_{1}$ within distance MinExistDist $r_{r_{2}}\left(r_{1}\right)$. The influence relation sets $n_{C} . S_{M}$, $n_{F} \cdot S_{M}, n_{M} \cdot S_{C}$, and $n_{M} . S_{F}$ can be determined with the following two theorems,

Theorem 1 Given $n_{C} \in L_{C}$, if $\exists n_{F} \in\left\{n_{F} \mid n_{M} \in n_{C} . S_{M}, n_{F} \in n_{M} . S_{F}\right\}$,

$$
\operatorname{MinDist}\left(r_{M}, r_{C}\right) \geq \operatorname{MinExistDist}{ }_{r_{F}}\left(r_{M}\right) \text {, }
$$

then for $m$ enclosed by $r_{M}, m$ is not influenced by any $c$ enclosed by $r_{C}$.

Proof We prove by contradiction. Suppose there is an $m$ enclosed by $r_{M}$ who is influenced by a specific $c$ enclosed by $r_{C}$, then according to Definition 2 , $d(c, m)<\operatorname{MinExistDist}_{r_{F}}\left(r_{M}\right)$; but since $d(c, m) \geq \operatorname{MinDist}\left(r_{M}, r_{C}\right)$ by the definition of MinDist, this contracts with $\operatorname{MinDist}\left(r_{M}, r_{C}\right) \geq \operatorname{MinExistDist}{ }_{r_{F}}\left(r_{M}\right)$.

Theorem 2 Given $n_{C} \in L_{C}$, if $\forall n_{F} \in\left\{n_{F} \mid n_{M} \in n_{C} . S_{M}, n_{F} \in n_{M} . S_{F}\right\}$,

$$
\operatorname{MaxDist}\left(r_{M}, r_{C}\right)<\operatorname{MinDist}\left(r_{M}, r_{F}\right),
$$

then for $m$ enclosed by $r_{M}$, for $c$ enclosed by $r_{C}, m$ is influenced by $c$.

Proof Since MaxDist $\left(r_{M}, r_{C}\right)<\operatorname{MinDist}\left(r_{M}, r_{F}\right)$, for any $m, c, f$ enclosed by $n_{M}, n_{C}$, and $n_{F}$, respectively, $d(m, c)<d(m, f)$. According to Definition $2, f$ enclosed by $n_{F}$ cannot influence $m$ enclosed by $n_{M}$ due to the existence of $n_{C}$. Also, since $n_{M} . S_{F}$ contains all $n_{F}$ s those could influence $n_{M}$ with the absence of $n_{C}, m$ enclosed by $n_{M}$ can only be influenced by some $c$ enclosed by $n_{C}$ when $c$ is added. 
Theorem 1 states that if node $n_{C}$ is so far away from node $n_{M}$ that there are other $n_{F} \mathrm{~s}$ much nearer to $n_{M}$, then customer $m$ s represented by $n_{M}$ are not influenced by any candidate location $c$ represented by $n_{C}$ due to the existence of $n_{F}$. To complete this intuition, Theorem 2 states that if node $n_{C}$ is so close to node $n_{M}$ that no other $n_{F} \mathrm{~s}$ can be closer, then customers represented by $n_{M}$ shall be influenced by some candidate location $c$ in $n_{C}$.

While Theorems 1 and 2 help determine the influence relations between node pairs, we need to compute and store further distance thresholds for node $n_{M} \in L_{M}$ to improve efficiency in determining influence relation sets for nodes in lists. Specifically, one of these thresholds, denoted as $n_{M} \cdot d_{l o w}$, stores the lower bound for the distance between $r_{M}$ and its nearest $r_{F}$, while the other, denoted as $n_{M} \cdot d_{u p p}$, stores the upper bound for the distance between $r_{M}$ and its nearest $r_{F}$. Formally, we have

$$
n_{M} \cdot d_{\text {low }}=\min \left(\left\{M i n \operatorname{Dist}\left(r_{M}, r_{F}\right) \mid \forall n_{F} \in n_{M} \cdot S_{F}\right\}\right)
$$

and

$$
n_{M} \cdot d_{\text {upp }}=\min \left(\left\{\text { MinExistDist } r_{F}\left(r_{M}\right) \mid \forall n_{F} \in n_{M} \cdot S_{F}\right\}\right) .
$$

With the theorems and stored distances introduced above, three rules are available for pruning and determining the influence relation set for child nodes $n_{M^{\prime}}$ of a given node $n_{M}$. The rules are as follows:

1. Given $n_{C}$, if $\exists n_{M} \in n_{C} . S_{M}, n_{M} . d_{l o w}>\operatorname{MaxDist}\left(r_{C}, r_{M}\right)$, then according to Theorem 2, $\forall m$ enclosed by $r_{M}$ and $\forall c$ enclosed by $r_{C}, m$ is influenced by $c$. Since we can ensure this customer in $r_{M}$ will be influenced, node $n_{M}$ should be removed from $n_{C} . S_{M}$ and $n_{C}$ should be removed from $n_{M} . S_{C}$ as well. In the meantime, for $c$ enclosed by $r_{C}, I_{C}^{l}$ should be increased by $\left|O_{M}\right|$.

2. Given $n_{C}$, if $\exists n_{M} \in n_{C} \cdot S_{M}, \operatorname{MinDist}\left(r_{M}, r_{C}\right) \geq n_{M} \cdot d_{u p p}$, according to Theorem 1, $\forall m$ enclosed by $r_{M}$ and $\forall c$ enclosed by $r_{C}, m$ is not influenced by $r_{C}$. Hence, $n_{M}$ and $n_{C}$ should be removed from $n_{C} \cdot S_{M}$ and $n_{M} \cdot S_{C}$, respectively.

3. Similar to rule 2, given $n_{F}$, if $\exists n_{M} \in n_{F} . S_{M}$, MinDist $\left(r_{M}, r_{F}\right) \geq n_{M} . d_{u p p}$, then $\forall m$ enclosed by $r_{M}$ and $\forall f$ enclosed by $r_{F}, m$ is not influenced by $f$. Thus, $n_{M}$ and $n_{F}$ should be removed from $n_{F} \cdot S_{M}$ and $n_{M} \cdot S_{F}$, respectively.

Fig. 6 shows an example of these pruning rules on influence relation sets. In this example, $n_{M} \cdot d_{u p p}=\operatorname{MinExistDist}\left(r_{M}, r_{F_{2}}\right), n_{M} \cdot d_{l o w}=\operatorname{MinDist}\left(r_{M}, r_{F_{1}}\right)$. According to rule $2, n_{C_{2}}$ is not in $n_{M} . S_{C}$ since $\operatorname{MinDist}\left(r_{C_{2}}, r_{M}\right) \geq n_{M} \cdot d_{u p p}$; according to rule $3, n_{F_{1}}$ is not in $n_{M} . S_{F}$ since $\operatorname{MinDist}\left(r_{F_{1}}, r_{M}\right) \geq n_{M} \cdot d_{u p p}$; according to rule 1 , any $c$ enclosed by $r_{C_{1}}$ should influence all $m$ enclosed by $r_{M}$, since $\operatorname{MaxDist}\left(r_{M}, r_{C_{1}}\right)<n_{M} \cdot d_{\text {low }}$.

Intuitively, the influence relation sets are initialized as the roots of corresponding trees, namely, $n_{\text {root }_{C}} . S_{M}=\left\{\operatorname{root}_{M}\right\}, n_{\text {root }_{F}} \cdot S_{M}=\left\{\operatorname{root}_{M}\right\}, n_{\text {root }_{M}} \cdot S_{C}=$ $\left\{\operatorname{root}_{F}\right\}$, and $n_{\text {root }_{M}} . S_{F}=\left\{\operatorname{root}_{F}\right\}$. As mentioned in algorithm 2, roots are stored in the lists with the influence relation information as entries. Each time an entry is processed, its child nodes are re-inserted into the corresponding lists 


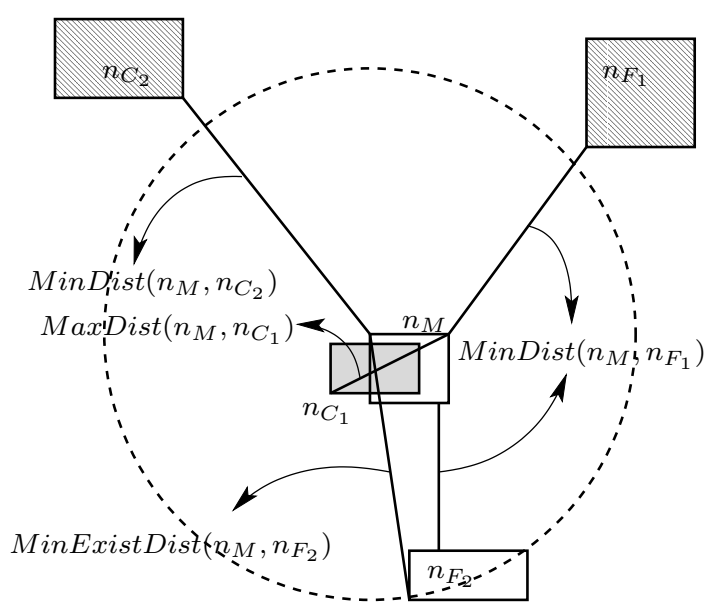

Fig. 6: $n_{F_{1}}$ can be pruned from $n_{M} . S_{F}, n_{C_{2}}$ can be pruned from $n_{M} \cdot S_{C}$

with their influence relation information computed. Before giving the details on how the entries are processed in Section 4.2.3, we will first introduce the criteria for determining the order of processing the entries in the next section.

\subsubsection{Importance of nodes}

We use the importance values of nodes to determine the access order for their corresponding entries in the lists. In EEP, different trees have different uses in pruning the search space: the candidate R-tree is traversed for the goal of computing influence value for candidates while the existing facility R-tree and the customer R-tree are traversed for the goal to prune unnecessary computations during computing the influence. Therefore, we have defined "importance" differently for different trees to guide the tree traversals towards different goals. To simplify the narration, we denote area $(n)$ and $|n|$ as the area of MBR and the number of locations enclosed in the MBR corresponding to node $n ;|n . S|$ as the number of nodes in $n$ 's influence relation set; n.imp is the importance value of node $n$.

- $n_{C} . i m p$. Since only the most influential candidates are desired in our problem, we want to always access the most promising candidates first. Recalling from Section 4.2.1, each entry in $L_{C}$ holds $I_{C}^{l}$ for node $n_{C}$ in it, we define the importance of a candidate node as the maximum number of customers it can influence estimated by influence relation information in that entry, i.e. $n_{C} . i m p=I_{C}^{l}+\sum_{n_{M} \in n_{C} . S_{M}}\left|n_{M}\right|$.

- $n_{F}$.imp. If the corresponding MBR of a facility node has a larger area, it may affect the estimation of the influence of more candidates. Also, a larger influence relation set on $M$ suggests a facility node being more relevant for costumers. Hence, $n_{F} . i m p$ is defined as area $\left(n_{F}\right) \cdot\left|n_{F} \cdot S_{M}\right|$. 
- $n_{M} . i m p$. Larger area $\left(o_{M}\right)$ and larger $\left|o_{M}\right|$ suggest a greater possibility for a customer node affecting the influence relation information of nodes in other lists, therefore the customer node is more useful for pruning the search space. With this rationale, $n_{M} . i m p$ is defined as area $\left(n_{M}\right) \cdot\left|n_{M}\right| \cdot$ $\left|n_{M} \cdot S_{F}\right| \cdot\left|n_{M} \cdot S_{C}\right|$.

\subsubsection{Processing entries}

As described in lines 4 to 6 in Algorithm 2 and the above sections, each time an entry with the greatest importance value on a list is retrieved, its child nodes are evaluated. If the node in the retrieved entry is a leaf node in $t_{C}$, denoted as $n_{C}$, then for $c$ in $n_{C}$ we compute their exact influence values by checking $I_{C}^{l}, n_{C} . S_{M}$ and $n_{M} . S_{F}$ for all $n_{M} \in n_{C} . S_{M}$. The computation is similar to the solution given in Section 4.1, however, here sets $M$ and $F$ are smaller thanks to stored influence relation information in the entry. If the node in the retrieved entry is an internal node, then we compute the influence relation information by inheriting it from the parent node and prune it using rules described in Section 4.2.1. Please note we can always inherit the information instead of computing it from scratch because the following property holds.

Property 1 For any child node $n_{M^{\prime}}$ of $n_{M}, n_{M^{\prime}} \cdot S_{F} \subset n_{M} \cdot S_{F}$, and $n_{M^{\prime}} \cdot S_{C} \subset$ $n_{M} . S_{F}$; for any child node $n_{C^{\prime}}$ of $n_{C}, n_{C^{\prime}} . S_{M} \subset n_{C} . S_{M}$; for any child node $n_{F^{\prime}}$ of $n_{F}, n_{F^{\prime}} . S_{M} \subset n_{F} . S_{M}$.

After obtaining the influence relation information for child node $n^{\prime}$, we should update the stored information in other lists related to the parent $n$. Again, we use the three rules based on distances between MBRs to prune unpromising nodes in the influence relation sets. If child node $n^{\prime}$ is still related to other nodes in the lists, then its importance value is computed and the node shall be re-inserted to the corresponding list. If the node retrieved is a leaf node in $t_{F}$ or $t_{M}$, we update its influence relation set, assign an importance value of -1 to it and re-insert it into list $L_{F}$ or $L_{M}$, respectively.

The algorithm terminates when there are at least $k$ computed candidate location cs' influence values and all nodes remaining in $L_{C}$ have maximum influence values smaller than the $k^{t h}$ largest influence values of candidates computed. The maximum influence values of $n_{C}$ in $L_{C}$ could be computed as $I_{C}^{u}=I_{C}^{l}+\left|n_{C} \cdot S_{M}\right|$. Also, when all entries on $L_{M}$ or $L_{F}$ have importance values smaller than 0 , Line 4 or Line 5 is omitted in Algorithm 2. Algorithm 3 gives the pseudo-code for the procedure in lines 4 to 6 of Algorithm 2. Note that in line 8 whether $n^{\prime}$ is related can be determined by checking the three rules introduced in Section 4.2.1.

\subsubsection{Complexity of EEP}

The construction of the R-tree indexes incurs $O(n \log n)$ cost. With the constructed R-tree, the major cost of EEP lies in the processing entry procedure, which computes the importance and influence relation information for children 


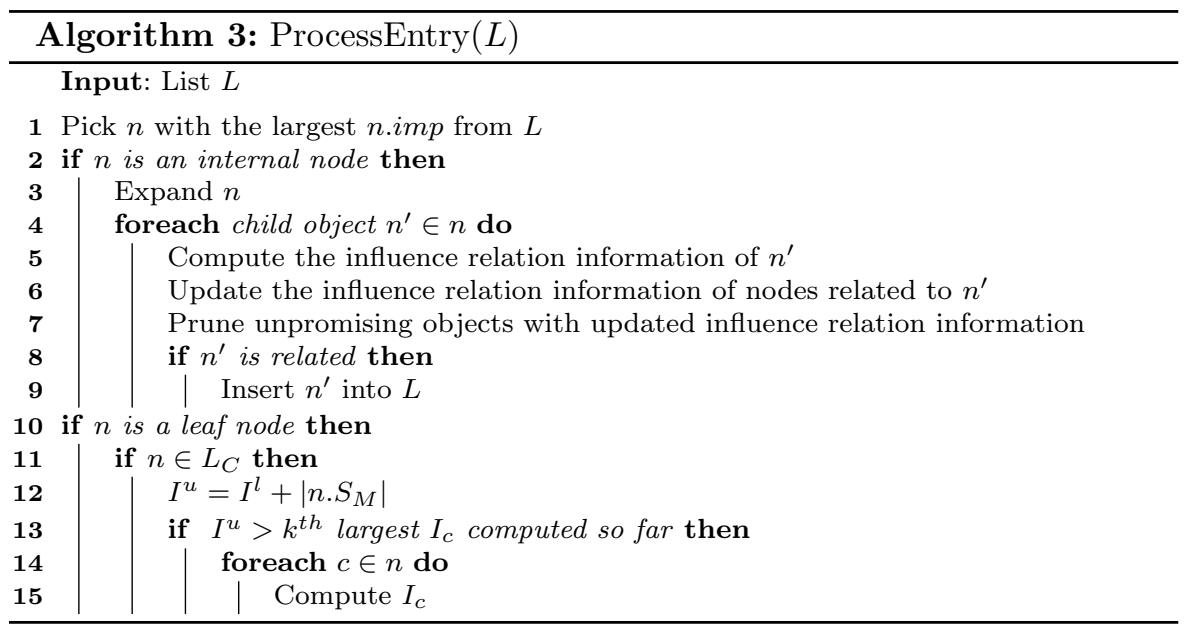

nodes of a node in the entry. Let $r$ denote the average size of the influence relation sets. As described in Algorithm 3, the cost of processing an entry is contributed by computing the influence relation information and updating influence relation information for other related nodes. Though in practical settings, $M$ tends to be much larger than both $F$ and $C$, we first denote their sizes as $O(n)$ for simplicity. For each entry, computing influence relation information costs $O(r)$ since a traversal on its parent's influence relation set is adequate. Updating influence relation information for related nodes is more complicated. For each entry on $L_{M}$, we need to check $n_{C} \in n_{M} . S_{C}$ and $n_{F} \in n_{M} . S_{F}$ to see whether they can be pruned, for $n_{C}$ updated, we also check $n_{M} \in n_{C} . S_{M}$ for further updates, resulting in $O\left(r^{2}\right)$ cost. For each entry on $L_{C}$, we need to check $n_{M} \in n_{C} . S_{M}$ to see whether $n_{M}$ can be pruned, also leading to an $O(r)$ cost. For each entry on $L_{F}$, we need to first check $n_{M} \in n_{F} . S_{M}$, for $n_{M}$ not pruned in this procedure, $n_{M} \cdot d_{\text {low }}$ and $n_{M} \cdot d_{\text {upp }}$ are updated. Additionally, we must evaluate $n_{C} \in n_{M} . S_{C}$ and $n_{F} \in n_{M} . S_{F}$ to see whether they can be pruned with the new distances. For $n_{C}$ updated, we check $n_{M} \in n_{C} \cdot S_{M}$ to further compute the influence upper bound for it. This procedure leads to a cost of $O\left(r^{3}\right)$.

In the best case, only $O(k)$ candidates with greatest influence values are accessed, therefore $O(k \cdot \log n)$ node $n_{C}$ s are accessed. The while loop of line 3 in Algorithm 2 only executes $O(\log n)$ times. Since influence relation sets are pruned smoothly, the relevant set size $O(r)=O(1)$. The overall cost of EEP in the best case is $O(n \log n)+O(\log n) \cdot\left(O(1)+O\left(1^{2}\right)+O(1)+O\left(1^{3}\right)\right)=$ $O(n \log n)$.

In the worst case, all candidates are accessed so the $t_{M}$ is actually also fully traversed. The while loop therefore executes $O(n)$ times. Here, the techniques eliminate few nodes from influence relation sets, thus $O(r)=O(n)$. To summarize, the overall cost of EEP in the worst case is $O(n \log n)+O(n)$. $\left(O(n)+O\left(n^{2}\right)+O(n)+O\left(n^{3}\right)\right)=O\left(n^{4}\right)$. 
Clearly, the major factor determining the efficiency of EEP is the average size $r$ of the influence relation sets. In Section 5 we will see that in both synthetic and real-world data sets, EEP is outperformed by SS in terms of both CPU time and the number of $\mathrm{I} / \mathrm{O}$ operations, indicating $r$ is close to $O(n)$. As previously mentioned, $|M|$ can be larger than $|F|$ and $|C|$ in reality. Thus if $|M|$ dominate the scale of the problem, the complexity of EEP can be represented by $O(|M| \log |M|)$ in the best case and $O\left(|M|^{3}\right)$ in the worst case.

\subsection{Bounding Influence Pruning}

EEP needs to maintain additional lists storing information of relation sets for not only nodes in the candidate tree $t_{C}$, but also for the customer tree $t_{M}$ and the existing facility tree $t_{F}$. This can consume a large amount of memory space when the data sets are huge. Also, as a result of maintaining influence relation lists for all data sets, its complexity in the worst case is at an undesired $O\left(n^{4}\right)$. Since we ultimately care only about most influential candidates in answering queries of the form defined in Definition 4 , it is desired to focus on the candidate tree $t_{C}$ rather than extensively studying all three trees.

In this section, we introduce another strategy to estimate and refine influence values for candidate locations without storing redundant information for customer locations and existing facility locations. Similar to EEP, we again index $C$ and $F$ with R-trees and $M$ with an aggregate R-tree.

Specifically, the method traverses $t_{C}$ in a best first order, using a max-heap to order currently available nodes by their influence upper bounds computed before their insertions. For each node on the heap, we store an influence region $n_{C} . R$, locating where all customers can only be influenced by the candidates in that node, a set $n_{C} . S_{F}$ of relevant $F$ node and a set $n_{C} . S_{M}$ of relevant $M$ node. A node $n_{F}$ is called relevant to $n_{C}$ if its existence potentially helps refining the influence regions for child node $n_{C^{\prime}}$ of $n_{C}$. A node $n_{M}$ is called relevant to $n_{C}$ if its MBR $r_{M}$ intersects the influence region $n_{C} . R$, namely, $m$ in $r_{M}$ might be influenced by $c$ in $r_{C}$.

Each time, the top node on the max-heap is retrieved. When the retrieved node is an internal one, the algorithm relies on geometric properties together with stored information in the retrieved node to refine its children nodes' influence regions and relevant sets. With this computed information, the influence upper bounds of child nodes can be retrieved by performing point enclosure queries on $t_{M}$. Next, each child node is evaluated with a global influence threshold, which is maintained globally as the $k^{\text {th }}$ greatest influence value of candidates computed so far. If it cannot be pruned, it is inserted into the max-heap for further consideration. When the retrieved node is a leaf node, the method uses further techniques to tighten the search space and performs exact influence computation for each location enclosed by the leaf node. Because the method focuses on bounding candidates and uses influence upper bounds to prune unpromising candidates, it's named the Bounding Influence 
Pruning (BIP) algorithm. Algorithm 4 lists the pseudo-code for BIP, where $I_{\delta}$ maintains the $k^{t h}$ maximum influence values seen so far.

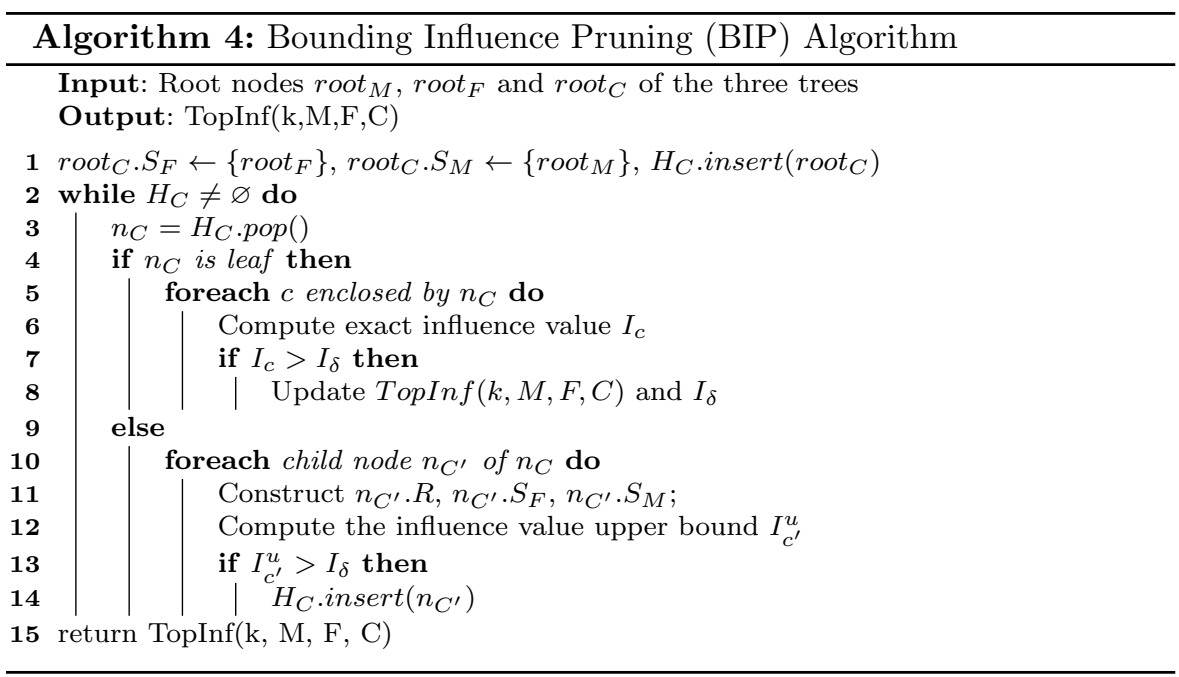

We will first introduce the methods for computing influence region and relevant sets (lines 11 to 12) in Section 4.3.1, and then elaborate on the techniques involved in computing exact influence values for candidates (line 6) in Section 4.3.2. The section ends with an analysis of the complexity of BIP in Section 4.3.3.

\subsubsection{Constructing influence region and relevant sets}

In this section, we describe the details for computing the influence region and relevant sets for a given internal node $n_{C}$. As described in Algorithm 4, the root nodes of $t_{M}$ and $t_{F}$ are inserted into the relevant sets root r. $_{S_{M}}$ and $\operatorname{root}_{C} . S_{F}$, respectively. Then, root $_{C}$ is inserted into the max-heap $H_{C}$.

Each time we pick the top node $n_{C}$ from $H_{C}$, which has the maximum $I_{C}^{u}$ among the nodes on the heap. To compute the influence region for a given node $n_{C}$, we first expand every $n_{F} \in n_{C} . S_{F}$ that has $r_{F}$ intersecting with $r_{C}$. Obviously, after a series of expanding operations, some $r_{F}$ will lie outside $r_{C}$ while other $r_{F}$ s will lie inside $r_{C}$. We call those $r_{F}$ s lying inside $r_{C}$ as inner relevant $F$ nodes, denoted as $r_{C} . S_{F}^{-}$. Since we only need to compute influence upper bound for $n_{C}$, we will only use $n_{C} . S_{F} \backslash r_{C} . S_{F}^{-}$to compute the influence region. For those customers locations laying inside $r_{C}$, we assume they are influenced by candidates in $n_{C}$ as we will ignore $n_{C} \cdot S_{F}^{-}$when computing the influence region. Fig. 7 shows an example of expanding $n_{F}$. In the figure, gray rectangles represent $n_{F}$ with $r_{F}$ intersecting with $r_{C}$, and black rectangles represent $n_{F} \in n_{C} . S_{F}^{-}$. 


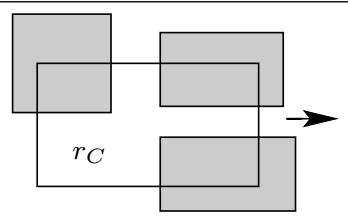

Step 0

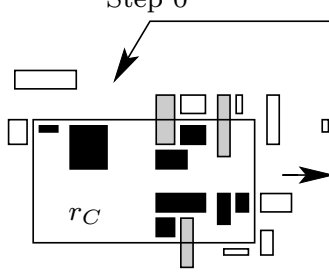

Step 3

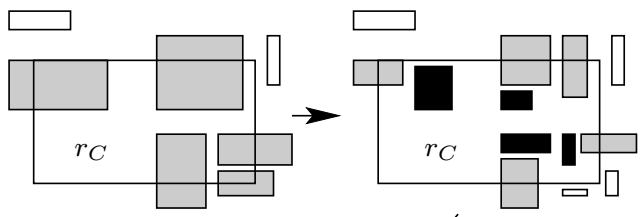

Step 1 Step 2

Fig. 7: Expanding $n_{F} \in n_{C} . S_{F}$ until no $n_{F}$ has $r_{F}$ intersecting with $r_{C}$

We use the following idea to compute the influence region for a given node $n_{C}$. On plane $\mathbb{P}$, given two points, their perpendicular bisector divides $\mathbb{P}$ into two regions, each of which contains one point. If another point $q$ is influenced by point $p_{1}$ rather than $p_{2}$, it must lie inside the half plane containing $p_{1}$ rather than that containing $p_{2}$. [6] generalizes this idea from points to rectangles. Given two rectangles $r_{1}$ and $r_{2}$, the generalized theorem uses multiple normalized perpendicular bisectors to divide the plane $\mathbb{P}$ into two regions, one of which contains all the points that might be influenced by points in $r_{1}$.

Formally, we introduce this idea using concepts antipodal corners, normalized perpendicular bisectors and Theorem 3 .

Definition 5 (Antipodal Corners) Let a rectangle $r$ 's lower left, lower right, upper left, and upper right corners be r.ll,r.lr, r.ul, r.ur, respectively. Given two rectangle $r_{1}$ and $r_{2}$, the antipodal corners of $r_{1}$ and $r_{2}$ are four pairs of corner points $\left(r_{1} . l l, r_{2} . u r\right),\left(r_{1} . l r, r_{2} . u l\right),\left(r_{1} . u l, r_{2} . l r\right)$, and $\left(r_{1} . u r, r_{2} . l l\right)$.

Definition 6 (Normalized Perpendicular Bisectors) Given two rectangles $r_{1}$ and $r_{2}$ and a pair of antipodal corners of $r_{1}$ and $r_{2},\left(a c_{1}, a c_{2}\right)$, the normalized perpendicular bisector of $a c_{1}, a c_{2}$, denoted as $N B_{a c_{1}, a c_{2}}$, is obtained through moving their perpendicular bisector, denoted as $B_{a c_{1}, a c_{2}}$, to intersect a point $p_{a c_{1}, a c_{2}}$, where

$$
\begin{aligned}
& p_{a c_{1}, a c_{2}} \cdot x= \begin{cases}\frac{r_{1} \cdot u r \cdot x+r_{2} \cdot u r \cdot x}{2}, & \text { if } a c_{1} \cdot x<a c_{2} \cdot x, \\
\frac{r_{1} \cdot u l \cdot x+r_{2} \cdot u l \cdot x}{2}, & \text { if } a c_{1} \cdot x \geq a c_{2} \cdot x .\end{cases} \\
& p_{a c_{1}, a c_{2}} \cdot y= \begin{cases}\frac{r_{1} \cdot u l \cdot y+r_{2} \cdot u l \cdot y}{2}, & \text { if } a c_{1} \cdot y<a c_{2} \cdot y \\
\frac{r_{1} \cdot l l \cdot y+r_{2} \cdot l l \cdot y}{2}, & \text { if } a c_{1} \cdot y \geq a c_{2} \cdot y .\end{cases}
\end{aligned}
$$

Fig. 8 shows an example of the normalized half planes described above.

The concepts defined above help us to find influence regions for a given node $n_{C}$. Specifically, perpendicular bisectors $a c_{1}$ and $a c_{2}$ divide the plane 


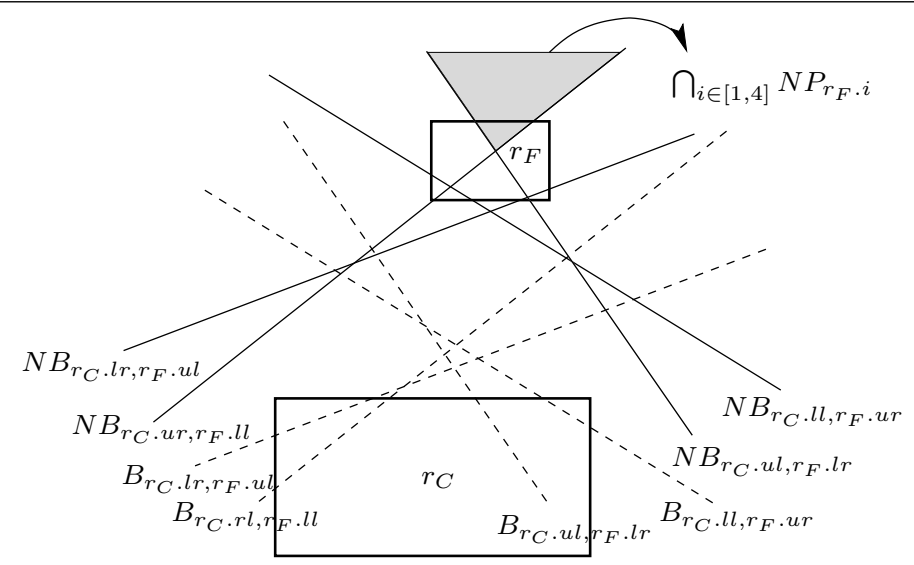

Fig. 8: Normalized half planes are divided by $N B_{r_{C} .1, r_{F} .3}, N B_{r_{C} .2, r_{F} .4}, N B_{r_{C} .3, r_{F} .1}, N B_{r_{C} .4, r_{F} .2} ; \quad$ the gray region $\bigcap_{i \in[1,4]} N P_{r_{F} . i}$ contains customers who won't be influenced by candidates enclosed by $r_{C}$ according to Theorem 3

$\mathbb{P}$ into two half planes, denoted as $P_{a c_{1}}$ and $P_{a c_{2}}$, respectively. The normalized perpendicular bisectors of $a c_{1}$ and $a c_{2}$ also divide the plane into two planes. Let $N P_{a c_{1}}$ be the normalized perpendicular bisector corresponding to the half plane $P_{a c_{1}}$ and $N P_{a c_{2}}$ be the one corresponding to the half plane $P_{a c_{2}}$. Remember that two rectangles have four pairs of antipodal corners, each pair of nodes contribute to a pair of normalized half planes. For brevity, let $r_{i} . l r=r_{i} .1, r_{i} . u r=r_{i} .2, r_{i} . u l=r_{i} .3, r_{i} . l l=r_{i} .4$, where $i=\{1,2\}$. Hence, the four pairs of half planes can be denoted as $\left(N P_{r_{1} .1}, N P_{r_{2} .3}\right),\left(N P_{r_{1} .2}, N P_{r_{2} .4}\right)$, $\left(N P_{r_{1} .3}, N P_{r_{2} .1}\right)$, and $\left(N P_{r_{1} .4}, N P_{r_{2} .2}\right)$. Given a node $n_{C}$ and a node $n_{F}$, and the theorem below proved by [6], any customer $m$ lying in $\bigcap_{i \in[1,4]} N P_{r_{2} . i}$ is not influenced by any candidate enclosed by $r_{C}$.

Theorem 3 Given two rectangles $r_{1}$ and $r_{2}$, let $p$ be a point in $\bigcap_{i \in[1,4]} N P_{r_{2} . i}$, where $\mathrm{NP}_{r_{2} . i}$ denotes a normalized half plane corresponding to $r_{2}$. Then the minimum distance between $p$ and any point in $r_{1}$ must be larger than the maximum distance between $p$ and any point in $r_{2}$.

The gray region in Fig. 8 is the described region of Theorem 3 of the given example. Since this region contains customers, it cannot be influenced by $n_{C}$, so we call it a pruning region on $n_{C}$ defined by $n_{F}$, denoted as $n_{C} \cdot P R_{n_{F}}$. The union of pruning regions defined by each $n_{F}$ in $F \backslash n_{C} . S_{F}^{-}$is a region containing customers not influenced. In other words, $\mathbb{P} \backslash \bigcap_{n_{F} \in F \backslash n_{C} \cdot S_{F}^{-}}\left(n_{C} \cdot P R_{n_{F}}\right)$ is a region containing customers potentially influenced by candidates enclosed by $r_{C}$ for a given $n_{C}$. We define this region as the influence region for $n_{C}$, i.e., $n_{C}$.R. However, according to its definition, computing this region would require extensive accesses on $F \backslash n_{C} . S_{F}^{-}$. To avoid this tremendous cost, we select the nearest $n_{F} \mathrm{~s}$ in 8 directions, denoted as $\operatorname{Western}(W)$, Southwestern $(S W)$, 
Southern(S), Southeastern(SE), Eastern(E), Northeastern(NE), Northern(N), Northwestern $(N W)$, of node $n_{C}$. Again, the distance indicator used is the maximum distance between two rectangles $r_{C}$ and $r_{F}$. Let $n_{C} \cdot N N_{n_{F}}$ denote the set for these eight nearest $n_{F} \mathrm{~s}, n_{C} \cdot R_{a p p}$ denote $\mathbb{P} \backslash \bigcap_{\left.n_{C} \cdot N N_{(} n_{F}\right)} n_{C} \cdot P R_{n_{F}}$, then since $n_{C} \cdot N N_{n_{F}} \subset F \backslash n_{C} \cdot S_{F}^{-}, n_{C} \cdot R \subset n_{C} \cdot R_{a p p}$. Region $n_{C} \cdot R_{a p p}$ is only an approximate influence region of $n_{C}$, but as it contains the desired $n_{C} . R$, it can be used as a substitute for computing the influence upper bound and relevant sets. In in the remainder of this paper, we use $n_{C} \cdot R$ to denote the approximate influence region if there is no ambiguity.

Fig. 9 demonstrates an example of computing $n_{C} \cdot R$ for a given $n_{C}$, where its nearest $8 n_{F} \mathrm{~S}$ are in different directions. In the figure, the nearest $n_{F} \mathrm{~s}$ are tagged by their corresponding direction to $n_{C}$. The inner polygon is $n_{C} . R$ is computed from $r_{C}$ and $r_{F}$ s using Theorem 3.

Due to the fact customers are indexed by an aggregate R-tree $t_{M} H$, we further bound the polygon $n_{C} \cdot R$ with several rectangles to enable efficient point enclosure queries on $t_{M}$. The gray region in Fig. 9 corresponds to these regions. In the implementation of the algorithm, it is these bounding rectangles rather than the exact $n_{C} . R$ that is used to compute $I_{C}^{u}$. Also, according to the definition of relevant $M$ set, only $n_{M}$ with $r_{m}$ lying inside $n_{C} \cdot R$ can have customers being influenced by $n_{C}$. For those intersecting $r_{M}$, we expand the corresponding $n_{M}$ just like expanding $n_{F}$ in the aforementioned description, until there is no intersection. Thus, $n_{C} \cdot S_{M}=\left\{n_{M} \subset n_{C} \cdot R\right\}$.

Besides obtaining the relevant $M$ set, we also need to compute the relevant $H F$ set with help from $n_{C}$.R. Recall that the operations in Theorem 3 divide the plane into 2 regions, one of which contains points not influenced by any point in $r_{1}$. We would like to use this to evaluate whether a node $n_{F} F \backslash$ $n_{C} . S_{F}^{-}$is relevant to $n_{C}$. Specifically, we apply Theorem 3 treating $n_{F}$ as the rectangle $r_{1}$, leading to a region $n_{F} \cdot P R_{n_{C}}=\bigcap_{i \in[1,4]} N P_{r_{C} . i}$ This $n_{F} \cdot P R_{n_{C}}$ contains customers that cannot be influenced by any existing facility locations $f$ enclosed by $r_{F}$. Obviously, if $n_{C} \cdot R \subset n_{F} \cdot P R_{n_{C}}$, then for any customer $m$ potentially influenced by $c$ in $n_{C}$, any $f$ enclosed by $r_{F}$ won't influence it. Namely, if a given node $n_{F}$ meets $n_{C} \cdot R \subset n_{F} \cdot P R_{n_{C}}$, it can be eliminated from $n_{C} . S_{F}$ since it is not relevant to the influence value of $n_{C}$. Of all nodes $n_{F}$ those are not eliminated by this evaluation form the outer relevant $F$ set of $n_{C}$, denoted as $n_{F} . S_{F}^{+}$. Then, we have $n_{C} \cdot S_{F}=n_{C} \cdot S_{F}^{+}+n_{C} \cdot S_{F}^{-}$. In Fig. 9, $f_{1}$ meets $n_{f_{1}} \cdot P R_{n_{C}} \subset n_{C} . R$ thus it will be eliminated from $n_{C} . S_{F}$, while $f_{2}$ has $n_{f_{2}} . P R_{n_{C}} \not \subset n_{C} \cdot R$, thus $f_{2} \in n_{C} . S_{F}$.

As described above, in order to compute $n_{C} . S_{F}^{+}$, we need to evaluate all $n_{F} F \backslash n_{C} . S_{F}^{-}$. This can be rather time-consuming when $F$ is massive. To overcome this, the following property is introduced,

Property 2 For child node $n_{C^{\prime}}$ of node $n_{C}$,

$$
n_{C^{\prime}} \cdot R \subset n_{C} \cdot R, n_{C^{\prime}} \cdot S_{F} \subset n_{C} \cdot S_{F}, n_{C^{\prime}} \cdot S_{M} \subset n_{C} \cdot S_{M} .
$$




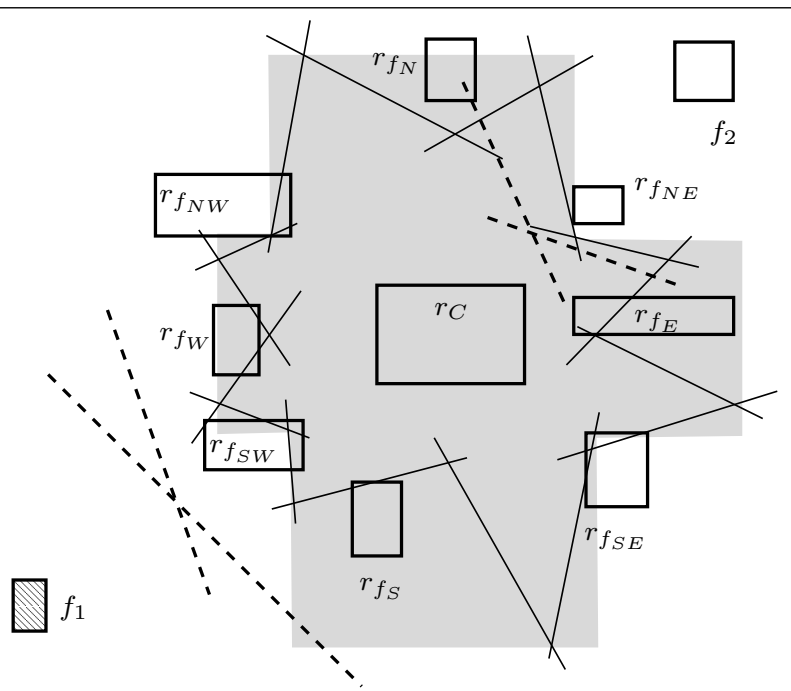

Fig. 9: Example of Influence Region

Property 2 enables us to tighten the search space for evaluating $n_{C} . S_{F}$. Let $n_{C^{\prime}}$ be a child node of $n_{C}$, when computing $n_{C^{\prime}} \cdot S_{F}^{+}$, instead of checking every $n_{F} \in F \backslash n_{C^{\prime}} . S_{F}^{-}$, it is adequate to check only $n_{F} \in n_{C} . S_{F} \backslash n_{C^{\prime}} . S_{F}^{-}$. This trick reduces unnecessary computation.

\subsubsection{Computing exact influence for candidates}

When the top node on max-heap $H_{C}$ is a leaf node, we need to compute the exact influence values for all candidate locations under it. Let's denote the retrieved leaf node as $n_{C}$. According to Property 2, we can compute influence regions and relevant sets for candidate location from $n_{C}$ 's relevant sets rather than from scratch. In other words, from the definition of relevant sets, $n_{C} \cdot S_{F}$ and $n_{C} \cdot S_{M}$ are the only customers and existing facilities that need to be considered in computing the influence value of $c \in n_{C}$, i.e. c. $S_{F} \subset n_{C} \cdot S_{F}$, c. $S_{M} \subset n_{C} . S_{M}$.

Naively, we could perform the sequential scan on relevant sets to obtain the exact influence value for $c$. However, thanks to the MBRs we stored in relevant sets, it is possible to further tighten the scan space with simple geometric checks. Again, the primary idea is that very distant customers are not likely to be influenced due to the existence of existing facilities near them. Also, very distant existing facilities cannot affect the influence value of a candidate since the customers can only be influenced by either nearby existing facilities or the candidate facility.

Formally, given candidate location $c$ under leaf node $n_{C}$, we first find 4 nearest $F$ nodes in the relevant $F$ set of $n_{C} . S_{F}$. Here, the distance criterion used is the maximum distance between any point in $r_{F}$ and $c$, denoted as MaxDist $\left(c, r_{F}\right)$. We use the furthest node to $c$ in each nearest $r_{F}$ to draw 


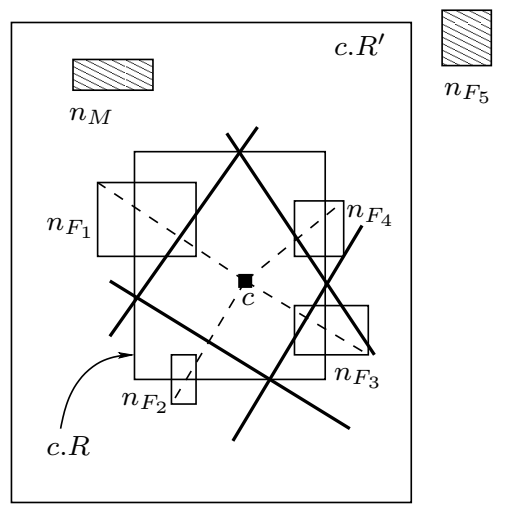

Fig. 10: $n_{F_{1-4}}$ are 4 nearest $n_{F} \in n_{C} . S_{F}, n_{M}$ can be pruned from $c . S_{M}$ since it lies outside $c . R, n_{F_{5}}$ can be pruned from $c . S_{F}$ since it lies outside $c . R^{\prime}$

a perpendicular bisector so together we have a Voronoi cell for $c$. Similar to the technique in [23], the Voronoi cell is bounded by a minimum rectangle denoted as c.R. According to the property of Voronoi cells, only customers located in this $c . R$ can be influenced by $c$. To prune relevant $F$ sets, we extend $c . R$ by doubling the distances between rectangle vertices and $c$, obtaining a new rectangle denoted as c. $R^{\prime}$. According to the conclusion in [23], all existing facilities outside this $c . R^{\prime}$ can be pruned when computing the influence of c. Using this properties, we have $c . S_{F}=\left\{n_{F} \in n_{C} . S_{F}, r_{F} \bigcap c . R^{\prime} \neq \emptyset\right\}$ and $c . S_{M}=\left\{n_{M} \in n_{C} . S_{M}, r_{M} \bigcap c . R \neq \emptyset\right\}$.

Fig. 10 gives an example of the technique described above, where $n_{F_{1-5}}$ are $5 F$ nodes in $n_{C} . S_{F}, n_{M}$ is a $M$ node in $n_{C} . S_{M}$. According to the property of bounding rectangles on Voronoi cells and the conclusion in [23], $n_{F_{5}}$ and $n_{M}$ can be pruned out from c. $S_{F}$ and c. $S_{M}$, respectively.

After $c . S_{F}$ and $c . S_{M}$ are refined, we can then employ sequential scan on them to obtain the exact influence value for $c$. However, since we still have $\mathrm{R}$-tree nodes in the relevant sets, it is possible to take further advantage of the property of MBRs before diving into distance computations between locations. Our basic idea is to avoid executing redundant distance computations for existing facility locations which simply give no helpful information. Specifically, given $c$ and a customer location $m$, we want to prune $n_{F}$ from $c . S_{F}$. To achieve this, we use a distance metric named MinMaxDist $\left(m, r_{F}\right)$, which was originally introduced in [20]. The MinMaxDist between a point $m$ and an MBR $r_{F}$ is defined as the minimum distance from $m$, within which at least one point enclosed in $r_{F}$ can be found. Note that according to the R-tree definition, there is at least one point on each edge of a given MBR. Therefore, we follow [31] to define the MinMaxDist as the $2^{\text {nd }}$ smallest distance among $d\left(m, f_{1-4}\right)$ where $f_{1-4}$ represent the four vertexes of $r_{F}$.

Together with the distance metric $\operatorname{MinDist}\left(m, r_{F}\right)$, we can obtain the following pruning rules. Given $m$ and $c$, 


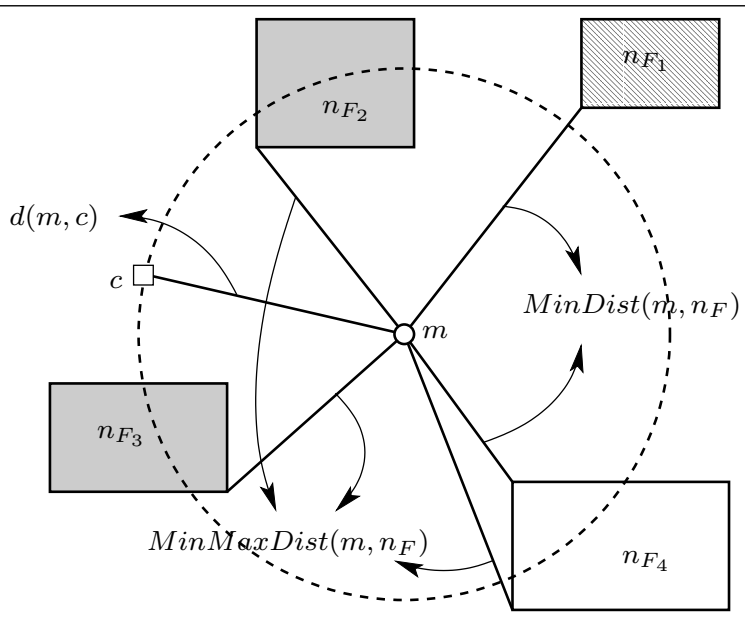

Fig. 11: $n_{F_{1}}$ can be discarded; $n_{F_{4}}$ suggests $c$ won't influence $m ; n_{F_{2}}$ and $n_{F_{3}}$ should be further expanded to help determining whether $c$ influences $m$

1. If for $n_{F} \in c . S_{F}, \operatorname{MinDist}\left(m, r_{F}\right) \geq d(c, m)$, then $n_{F}$ can be pruned from c. $S_{F}$; This is because if $\operatorname{MinDist}\left(m, r_{F}\right) \geq d(c, m), \forall f \in r_{F}, d(f, m) \geq$ $d(c, m)$, i.e., whether $c$ influences $m$ is irrelevant to $r_{F}$;

2. If for $n_{F} \in c . S_{F}, \operatorname{MinMaxDist}\left(m, r_{F}\right)<d(c, m), c$ cannot influence $m$; This is because according to definition of MinMaxDist, $\exists f \in r_{F}$ that $d(f, m)<d(c, m)$

3. If for $n_{F} \in c . S_{F}, \operatorname{MinDist}\left(m, r_{F}\right)<d(c, m)$ and $\operatorname{MinMaxDist}\left(m, r_{F}\right) \geq$ $d(c, m), n_{F}$ should be preserved in $c . S_{F}$ for further computation; Since we are not sure whether there is a $f \in r_{F}$ that $\operatorname{MinDist}\left(m, r_{F}\right)<d(f, m)<$ MinMaxDist $\left(m, r_{F}\right)$, we are not sure whether $c$ can influence $m$ given $r_{f}$.

Fig. 11 gives an example of the above pruning rules. On determining whether a given $c$ influences $m, n_{F_{1}}$ should be discarded since it provides no information for the computation; $n_{F_{4}}$ should be preserved in $c . S_{F}$ for further computations; and if either $n_{F_{2}}$ or $n_{F_{3}}$ is present, then we can be sure $c$ cannot influence $m$, so we can skip checking this $m$.

\subsubsection{Complexity of BIP}

The computational cost of BIP can be divided into index construction, pruning and exact influence computation. The R-tree indexes can be constructed in $O(n \log n)$ cost.

In the best case, only $O(k)$ most influential candidates are accessed. Thus only $k O(\log n)$ nodes $n_{C}$ in $t_{C}$ is accessed. This is because if the pruning technique works well, most of the irrelevant $F$ and $M$ locations are pruned after the construction of the influence region and relevant sets in the beginning with $O(n)$ cost. Thanks to Property 2, subsequent computations on influence upper bounds and relevant sets consume $O(1)$. Also, the exact influence computation 
procedure only need to consume $O(k) \cdot O\left(c . S_{M}\right) \cdot O\left(c . S_{F}\right)=O(k)$. Thus, the overall run-time complexity of BIP in the best case is $O(n \log n)+O(n)+$ $O(k)=O(n \log n)$.

In the worst case, all candidates are accessed, therefore all nodes in $t_{C}$ are accessed. For each accessed node $n_{C}$ in $t_{C}$, an $O(n)$ cost is needed to compute the influence upper bound and relevant sets. Also, an $O(n)$ cost is again needed for further pruning of c. $S_{M}$ and $c . S_{F}$ before computing the exact influence. Due to ineffectiveness of pruning, a cost of $O(n) \cdot O(n) \cdot O(n)=O\left(n^{3}\right)$ is needed when computing the exact influence values. To summarize, the overall run-time complexity of BIP in the worst case is $O(n \log n)+O\left(n^{2}\right)+O\left(n^{3}\right)=O\left(n^{3}\right)$.

That is to say, the bottleneck of BIP could be the exact influence value computation since it simply follows a three-layer loop on all three data sets. Yet as demonstrated in Section 5, BIP beats SS, which is of a $O\left(n^{2}\right)$ complexity, in terms of CPU time. This suggests that in both synthetic and real world data sets, BIP can offer a reasonable pruning effect, substantially eliminating unnecessary computations during the traversal.

Similar to the analysis for EEP, when we perceive $|M|$ as the dominating data set in the input, the complexity of BIP can be represented by $O(|M| \log |M|)$ in both the best case and the worst case. This is because BIP centers on candidate tree $t_{C}$ and tries to avoids repetitive access on $M$. Even in the worst case, the pruning phase costs $O(|M|)$ and exact influence computation also only costs $O(|M|)$, leaving the overall cost to be $O(|M| \log |M|)$.

\subsection{Nearest Facility Circle Join}

EEP and BIP have $O\left(n^{4}\right)$ and $O\left(n^{3}\right)$ worst-case complexity due to a large number of traversals on $t_{F}\left(t_{M}\right)$. To achieve lower worst-case complexity, we propose a new algorithm named Nearest Facility Circle Join (NFCJ), which avoids repetitive accesses by pre-computing and indexing the influence relationship. Similar to the sequential scan, NFCJ involves two steps: 1) computing influence relationship between the existing facilities and customers; 2) computing influence values for candidates. The difference between NFCJ and the sequential scan is that NFCJ leverages spatial indexes to enhance the efficiency of both steps. Specifically, in the first step, the nearest facility circles for all customers are computed using all nearest neighbor algorithms (e.g., [27] [35]), and are indexed in an R-tree, denoted as the influence R-tree. In the second step, this influence R-tree is queried to find which nearest facility circles a candidate location lies in. Here, the number of nearest facility circles a candidate lies in is essentially the influence value of that candidate location. We further improve the efficiency of the algorithm by indexing the candidates with an R-tree and querying the influence R-tree for candidates together. Then the querying process effectively becomes an R-tree intersection join. This way, repetitive traversals in EEP and BIP are avoided. We present the details of NFCJ as follows. 


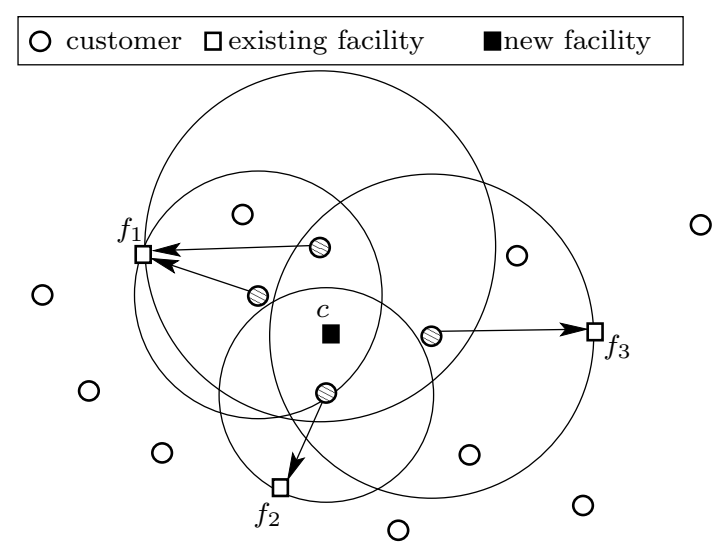

Fig. 12: $c$ lies in 4 nearest facility circles of customers(shaded ones), therefore $I_{c}=4$

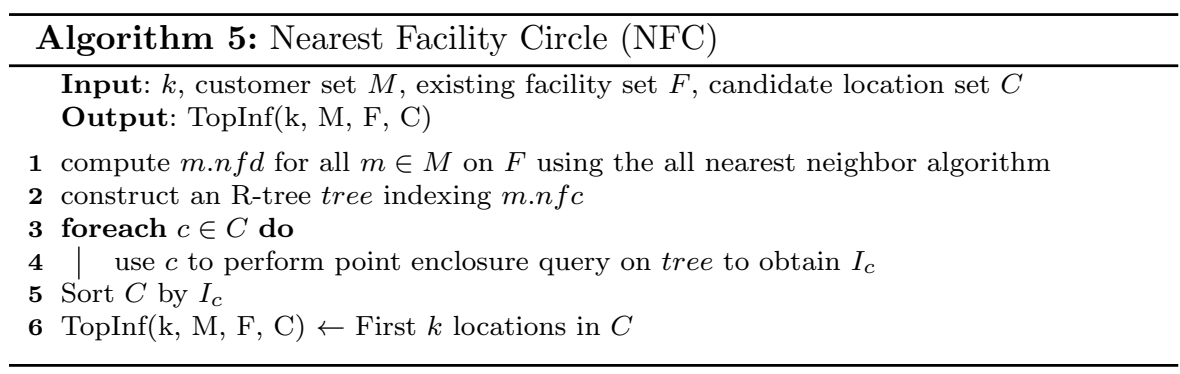

In the first step, we need to compute the nearest facility circle for each customer location. The nearest facility circle of a customer $m$ is a circle centered at $m$, with a radius of $\min _{\forall f \in F}(d(f, m))$. To find the nearest facility circle for all customer locations, we can leverage all nearest neighbor search algorithms, where the task is to find the nearest neighbors for all data points. With these circles, a new facility would be the nearest facility of a given customer $m$ if and only if it located in the nearest facility circle of $m$. That is to say, the potential influence of a candidate location can be computed as the number of the nearest facility circles the candidate lies in. To easily determine which circles a candidate location lies in, we construct an R-tree on the nearest facility circles and denote this tree as the influence R-tree. Naively, the influence value of a candidate location can be computed by a point enclosure query on the influence R-tree using the candidate location as the query point. For example, in Fig. 12, the new facility $c$ is located in four nearest facility circles (corresponding to the four shaded customers), therefore its potential influence is 4. This algorithm enhances only the first step,i.e., computing the nearest facility circles for customers, and is named Nearest Facility Circle (NFC). Its pseudo-code is shown in Algorithm 5. 


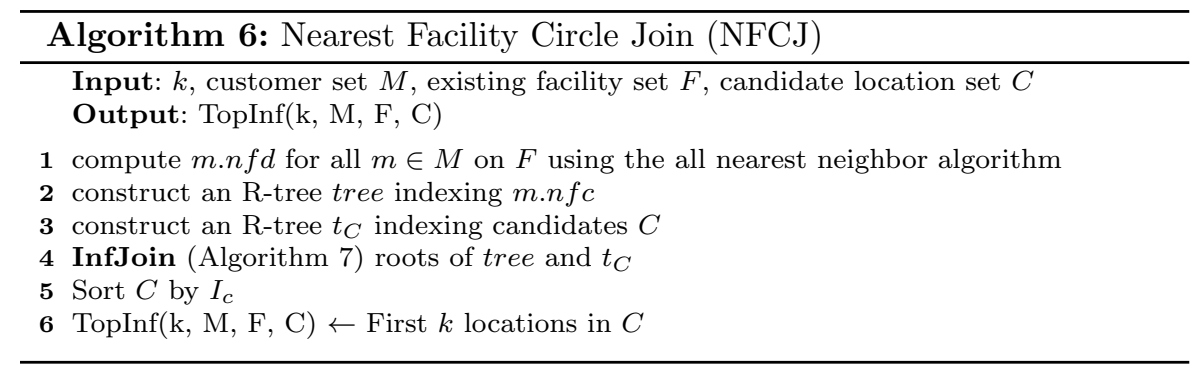

It is easy to observe that in NFC algorithm, when computing the influence in the second step, the candidates are considered individually, which does not benefit from batch processing on the large candidate set. We propose to construct another R-tree on the candidate set such that the influence computation step can be done via an intersection join between this candidate $\mathrm{R}$-tree and the influence R-tree. The expected improvement brought by the additional R-tree will be the competitive CPU time and much less I/O operations as the query procedure is done for a group of candidates altogether. We denote this algorithm as Nearest Facility Circle Join (NFCJ). Algorithm 6 shows the steps of NFCJ, where the point enclosure query in NFC is replaced by an intersection join procedure (line 4) over the two R-trees [4]. We describe the details of the join procedure as follows.

The join procedure begins from the roots of two trees and recursively calls a method (shown in Algorithm 7) to join the child nodes of two given nodes from the candidate R-tree and the influence R-tree, respectively. Here, two nodes should be joined together if their MBRs intersect each other. If both two to-be joined nodes are internal nodes, a plane sweep procedure is used to determine which child nodes should be joined (lines 1 to 2 ). This plane sweep procedure first sorts the child nodes of each node by their MBR coordinates respectively, and then checks the intersection relationship between nodes in the two sorted queues. If either node is leaf and the other is internal, then the child nodes of the internal node are joined with the leaf node if they intersect the leaf node (lines 3 to 10). If both nodes are leaf nodes, the number of nearest facility circles joined with that candidate is added to a counter corresponding that candidate (lines 11 to 13). After all leaf nodes in the candidate R-tree are joined with the intersecting nearest facility circles, the counter of each candidate gives the influence value of that candidate.

We analyze the complexity of NFCJ algorithm as follows. For the first step, algorithms such as the k-d tree based nearest neighbor search algorithm and the Voronoi diagram algorithm can solve the all nearest neighbor problem on the plane with cost of $O(n \log n)$ [27] [35]. Thus, the nearest facility circles can be computed with cost of $O(n \log n)$. The construction of an R-tree index tree takes $O(n \log n)$ as well. For the second step, the R-tree join algorithm achieves at best $O(n \log n)$ and at worst $O\left(n^{2}\right)$ run-time performance. To summarize, the run-time complexity of NFCJ algorithm is $O(n \log n)$ in the best case 


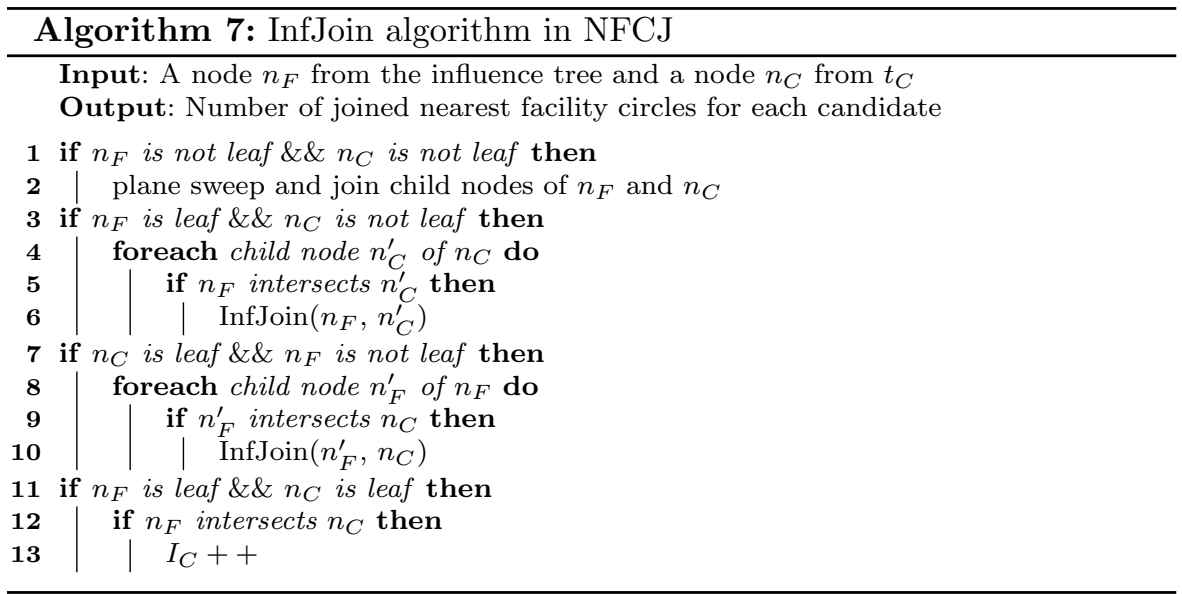

and $O\left(n^{2}\right)$ in the worst case. In practical situations, where the customer data set is likely to be much larger than both the facility and the candidate data sets, the most expensive parts in NFCJ are computing the nearest facilities for every customer and constructing an R-tree on the nearest facility circles, both of which incur $O(n \log n)$ cost. In other words, when $|M|$ dominates the problem scale, the overall cost of NFCJ is $O(|M| \log |M|)+O(\log |M|)=$ $O(|M| \log |M|)$. This explains the advantages that NFCJ maintains over EEP and BIP since NFCJ manages to achieve an $O(|M| \log |M|)$ complexity with much less tree traversals than EEP and BIP.

\section{Performance Study}

In this section, we present a performance study conducted on the algorithms described in Section 4. Notice that we are dealing with two dimensional space data sets, and we use two double type variables to store a location. Hence the biggest data set used in the experiment, namely the $4 M$ data set of $M$, only consumes $64 \mathrm{MB}$ main memory, which is negligible for a modern computer. In the remainder of this section, we still show results on both CPU time and I/O operation number for the sake of completeness. Additionally, to follow the tradition in the literature and to conduct a comparison of the true performance, we do not use any page buffering or cache scheduling techniques in our experiments. We incorporate the I/O operation cost into the CPU time cost to roughly compute a running time cost (by timing a $12 \mathrm{~ms}$ random read latency of hard disk to the number of I/O operations) for EEP, BIP, NFC, and NFCJ. NFC is included in all experiments to show the effectiveness of the join procedure in NFCJ. 


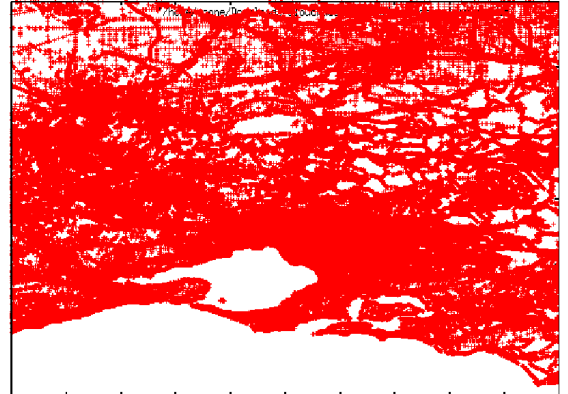

(a) Melbourne data sets

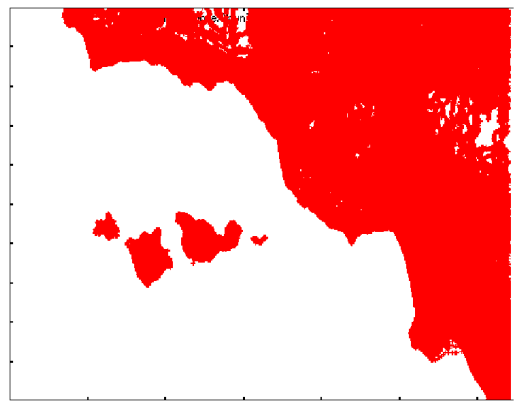

(b) Los Angeles data sets

Fig. 13: Real world data sets distribution

\subsection{Experimental Setup}

We conduct all experiments on a workstation running CentOS 6.4 with a 3.2 GHz six-core CPU and 6GB RAM memory. All algorithms are implemented in $\mathrm{C}++$. Specifically, the implementation of the NFC and NFCJ algorithm used the methods of [2] to perform all nearest neighbor queries.

Both synthetic and real world data sets are used in our experiments. The synthetic data sets are generated to contain 20 clusters; each cluster in the Gaussian data sets and the Zipfian data sets follows Gaussian and Zipfian distribution, respectively. The real world data sets contain 1,758,928 and 2,330,014 real place locations in Melbourne, VIC, Australia and Los Angeles, CA, United States, respectively. This data are part of OpenStreet Map Project [18] and are pre-processed by CloudMade [10]. The distribution of the real world data sets are shown in Fig. 13. For all data sets, we uniformly sample specific number of data points to form separate sets $C, F$, and $M$.

The parameters and data set cardinalities are listed in Table 3, where the default values are highlighted in bold style.

Table 3: Experiment configurations

\begin{tabular}{l|l|l|l}
\hline Parameter & Synthetic Data & Melbourne & Loa Angeles \\
\hline \hline$|M|$ & $500 \mathrm{~K}, 1 \mathrm{M}, \mathbf{2 M}, 4 \mathrm{M}$ & $200 \mathrm{~K}, 400 \mathrm{~K}, \mathbf{8 0 0 K}, 1.6 \mathrm{M}$ & $250 \mathrm{~K}, 500 \mathrm{~K}, \mathbf{1 M}, 2 \mathrm{M}$ \\
\hline$|F|$ & $5 \mathrm{~K}, \mathbf{1 0 K}, 20 \mathrm{~K}, 40 \mathrm{~K}$ & $2.5 \mathrm{~K}, \mathbf{5 K}, 10 \mathrm{~K}, 20 \mathrm{~K}$ & $5 \mathrm{~K}, \mathbf{1 0 K}, 20 \mathrm{~K}, 40 \mathrm{~K}$ \\
\hline$|C|$ & $25 \mathrm{~K}, \mathbf{5 0 K}, 100 \mathrm{~K}, 200 \mathrm{~K}$ & $10 \mathrm{~K}, 20 \mathrm{~K} \mathbf{4 0 K}, 80 \mathrm{~K}$ & $25 \mathrm{~K}, \mathbf{5 0 K}, 100 \mathrm{~K}, 200 \mathrm{~K}$ \\
\hline$k$ & $1, \mathbf{1 0}, 100,1000,10000$ & $\mathbf{1 0}$ & $1, \mathbf{1 0}, 100,1000,10000$ \\
\hline Node Size & $1 \mathrm{~K}, \mathbf{2 K}, 4 \mathrm{~K}$ & $\mathbf{2 K}$ & $1 \mathrm{~K}, \mathbf{2 K}, 4 \mathrm{~K}$ \\
\hline Distribution & Gaussian, Zipfian & Real & Real \\
\hline
\end{tabular}

5.2 Effect of Parameter $k$

As different values of parameter $k$ are expected to affect the pruning power of the proposed branch-and-bound algorithms BIP and EEP, we conduct experiments using default configuration. Fig. 14 gives the results of the performance 


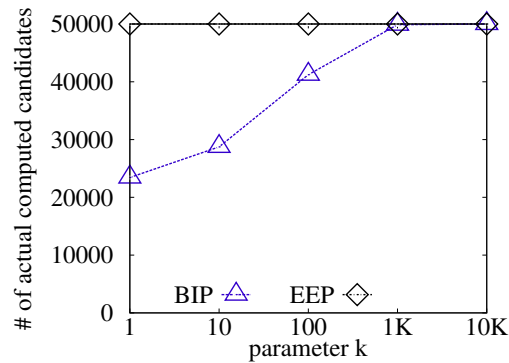

(a) \# of computed candidates, Zipfian

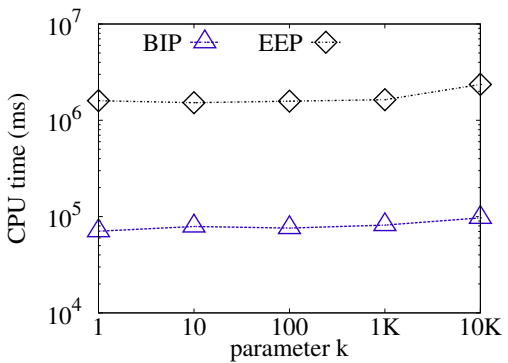

(b) CPU time, Zipfian

Fig. 14: Effect of parmeter $k$

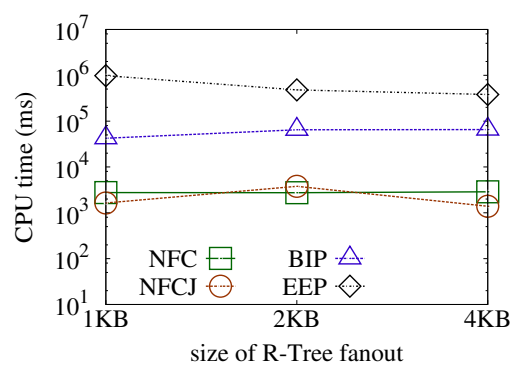

(a) CPU time, Los Angeles

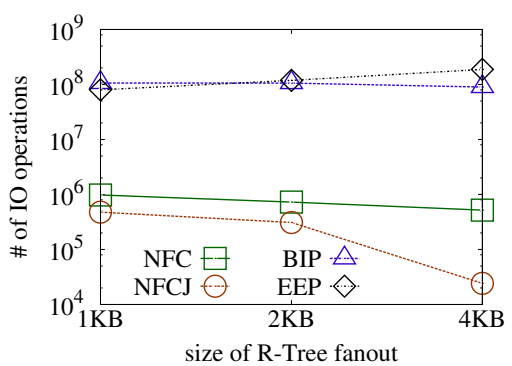

(b) \# of I/O, Los Angeles

Fig. 15: Effect of fanout

of BIP and EEP when varying $k$ from 1 to 10000 on the Zipfian data sets with the default setting $(|C|=50 \mathrm{~K})$. Results on other data sets are similar thus omitted here. Though the two algorithms are designed to leverage the parameter $k$ to early terminate as described in Section 4.2 and Section 4.3, it turns out that in most cases their pruning power is limited on the candidate sets. While BIP can prune around half of the candidates when $k$ is small, EEP can rarely prune any in our experiments. This can be explained by two reasons. First, during the procedure of pruning in both algorithms, all three $\mathrm{R}$-trees are involved; as each R-tree bounds the branch by aggregating the spatial information, involving all of them renders the obtained bounds vastly less effective. Second, both branch-and-bound algorithms aim at pruning the existing facility and the customer set in addition to the candidate set; the less effectiveness on the candidate sets may not reflect the pruning power of the algorithms on the other two sets. As the result, the performance of the algorithms in terms of CPU time is relatively insensitive towards the value of $k$, which is demonstrated in Fig. 14b.

\subsection{Effect of Node Size (fanout)}

We vary the R-tree node size to study its effect on the performance of the proposed algorithms. The corresponding results on the Los Angeles data sets are shown in Fig. 15. As demonstrated in these figures, in all cases, both NFC 
and NFCJ significantly outperforms EEP and BIP in two orders of magnitude. While the increased size of node does decrease the I/O performance of both NFC and NFCJ gradually, it has little effect on the CPU time cost of NFC and NFCJ. This is because for the same amount of data, bigger nodes lead shorter trees but do not bring significant benefits on distance computation. Both EEP and BIP are less sensitive towards the variation of node size as the variation here is not significant, it may take a much larger variation on the tree height to affect the performance of branch-and-bound algorithms. It can also be observed that when the node size is $2 K$, the CPU time of NFCJ turns out to be outperformed by NFC; this can be explained by that the performance of $\mathrm{R}$-tree join is highly dependent upon the (spatial) grouping arrangement of Rtree, when the node size is set to $2 K$, the overlapping regions between different branches in the candidate R-tree could be relatively large that the efficiency earned by grouping query is overweighted by traversing multiple branches of one tree for every node in the other tree.

\subsection{Effect of Data Set Cardinality}

In this section, we study the effect of the cardinality of each data set has on all algorithms. For each case, the CPU time, the number of I/O operations and the running time derived by summing the I/O cost (timed by $12 \mathrm{~ms}$ latency per operation) and the CPU time cost are shown respectively for all algorithms.

Effect of candidate cardinality $|C|$. It is expected that more candidates result to higher query cost. We vary the cardinality of candidate set and list the performance of all algorithms on four data sets in Fig. 16. Overall, the performance of all algorithms degrades gradually as the cardinality of $C$ grows. NFC and NFCJ outperform all other algorithm to up two, four, and three orders of magnitudes in terms of the CPU time, the number of I/O operations, and the running time, respectively. EEP turns out to be less efficient than SS. The reason for this is that EEP traverses the tree guided by the importance of nodes; it may follow a traversal order more like breadth first search such that it degrades to SS at the bottom of the trees but has additional overhead on attempt of pruning. This suggests that the performance of EEP tends to have a time complexity close to that in the worst case. BIP outperforms SS by almost one order of magnitude in terms of CPU time. The reason behind this is that BIP follows a best first search more like a depth-first search, i.e., computing the exact influence values for part of the candidates first and use them as the guide during traversal. The overall performance result fit to our theoretic analysis that NFC and NFCJ have the lower time complexity comparing to both BIP and EEP. Additionally, in terms of number of operations, NFCJ constantly beats NFC by several times, justifying the intuition of grouping point enclosure queries by using the candidate R-tree. This advantage makes NFCJ the best performing algorithm in terms of running time cost.

Effect of facility cardinality $|F|$. The cardinality of the facility set only has a linear effect on the performance when the sequential scan method is 


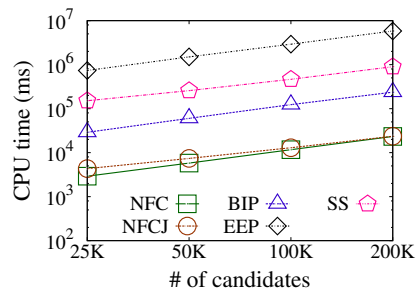

(a) CPU time, Gaussian

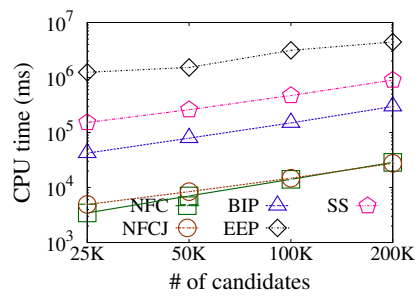

(d) CPU time, Zipfian

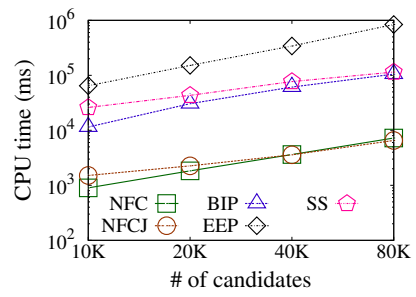

(g) CPU time, Melbourne

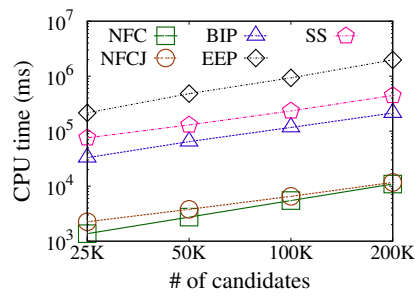

(j) CPU time, Los Angeles

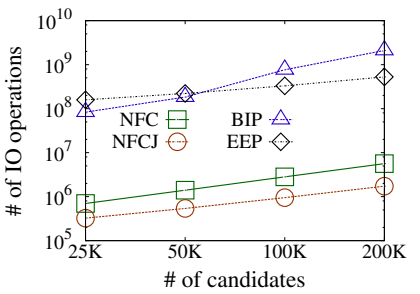

(b) \# of I/O, Gaussian

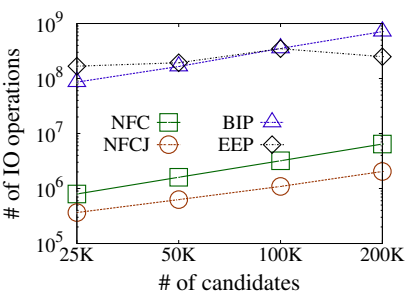

(e) \# of I/O, Zipfian

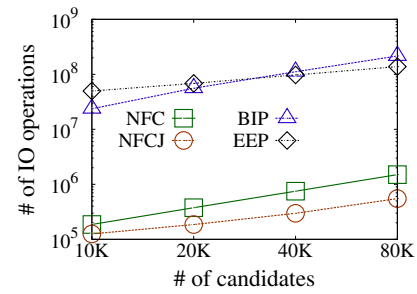

(h) \# of I/O, Melbourne

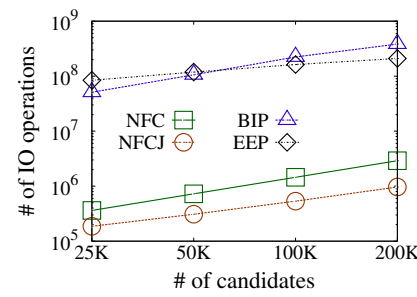

(k) \# of I/O, Los Angeles

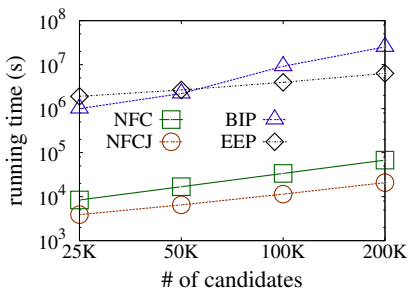

(c) Running time, Gaussian

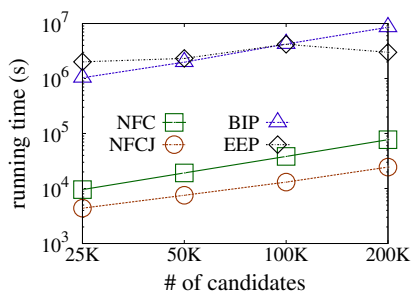

(f) Running time, Zipfian

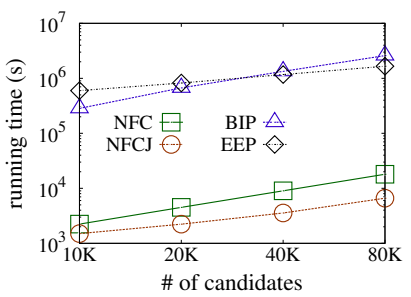

(i) Running time, Melbourne

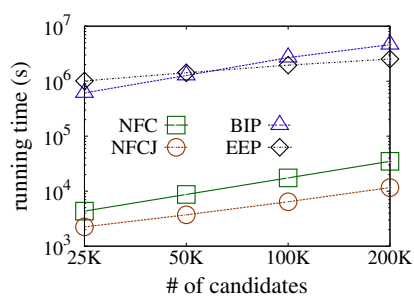

(l) Running time, Los Angeles

Fig. 16: Effect of the cardinality of the candidate set $C$

used. However, it may have significant effect on algorithms that utilize the facility set to prune the computations. We vary this cardinality and show the results of the performance of all algorithms in Fig. 17. As illustrated in the figures, the performance of BIP and NFC is improved while that of NFCJ stays the same when the number of existing facilities grows. For BIP, the effect on $\mathrm{CPU}$ time is two-edge: the more existing facilities, the smaller the influence region for each candidate node, yet also the more facilities to be evaluated to see whether they are relevant. These two factors explain the first decreasing then increasing CPU time cost in Fig. 17a and Fig. 17d, and also the slightly 


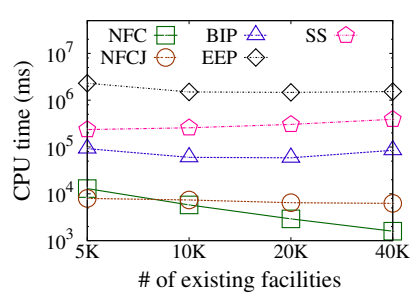

(a) CPU time, Gaussian

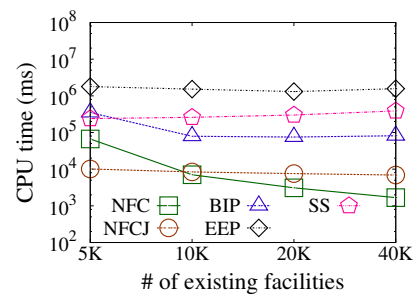

(d) CPU time, Zipfian

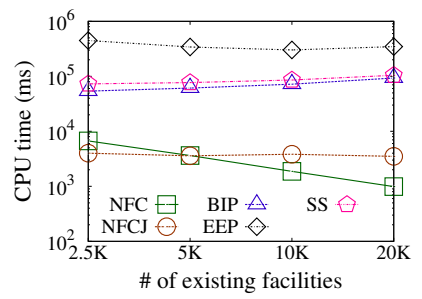

(g) CPU time, Melbourne

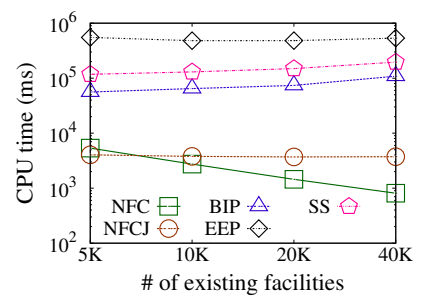

(j) CPU time, Los Angeles

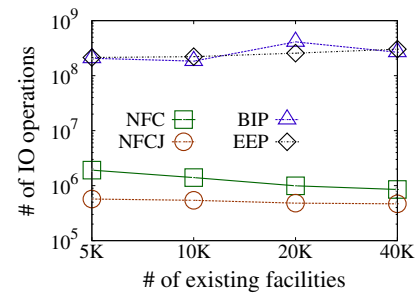

(b) \# of I/O, Gaussian

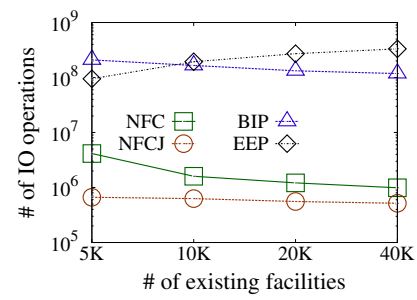

(e) \# of I/O, Zipfian

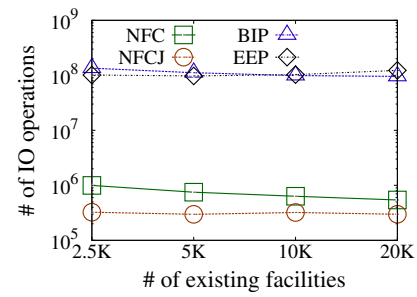

(h) \# of I/O, Melbourne

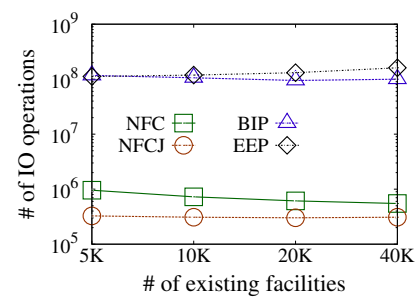

(k) \# of I/O, Los Angeles

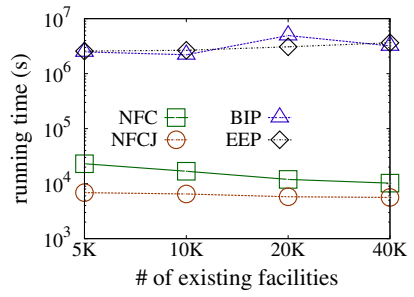

(c) Running time, Gaussian

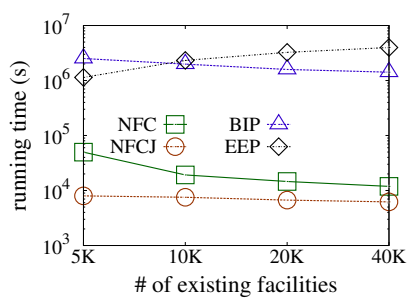

(f) Running time, Zipfian

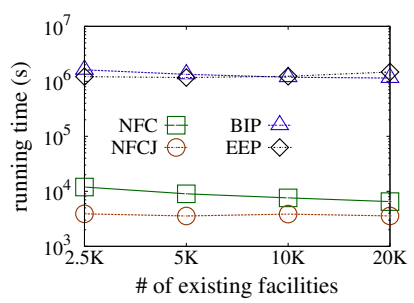

(i) Running time, Melbourne

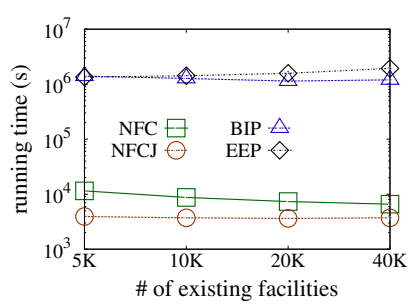

(1) Running time, Los Angeles

Fig. 17: Effect of the cardinality of the existing facility set $F$

increasing CPU time cost in Fig. 17g and Fig. 17j. However, the benefit of more existing facilities, i.e., the greater pruning power, does gain advantage in terms of the number of I/O operations, as depicted in Fig. 17b, Fig. 17e, Fig. 17h, and Fig. 17k. For NFC, the more existing facilities indicate the smaller each nearest facility circle will be, which result to the better organized $\mathrm{R}$-tree as less nearest facility circles are likely to overlap with each other. This is why NFC enjoys the greater existing facility set $F$ in all cases shown in the figures. However, NFCJ is not sensitive towards the varying $F$ as it groups candidates and nearest facility circles together and traverse both trees level by 


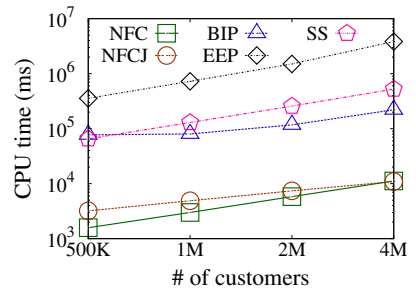

(a) CPU time, Gaussian

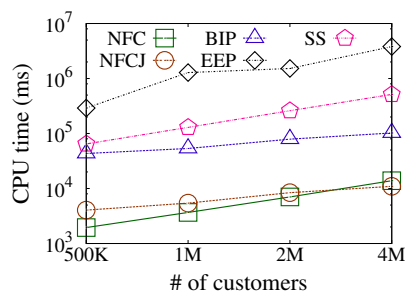

(d) CPU time, Zipfian

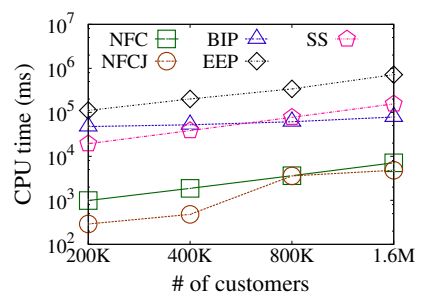

(g) CPU time, Melbourne

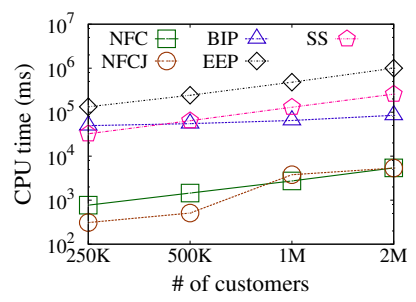

(j) CPU time, Los Angeles

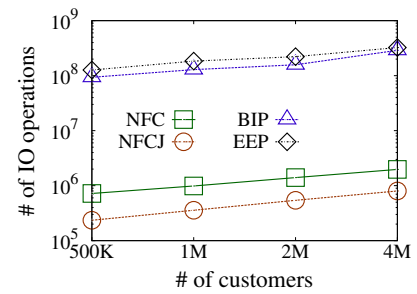

(b) \# of I/O, Gaussian

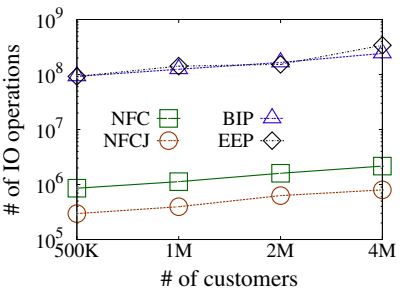

(e) \# of I/O, Zipfian

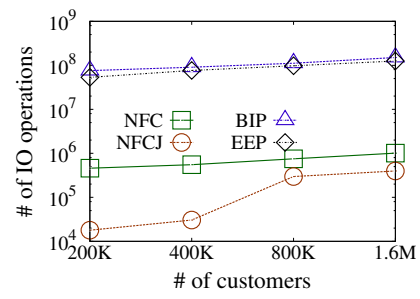

(h) \# of I/O, Melbourne

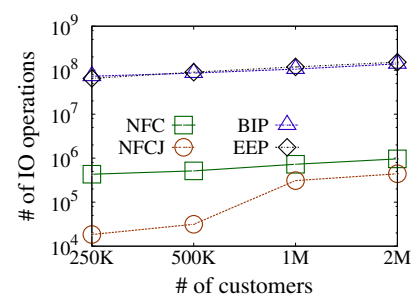

(k) \# of I/O, Los Angeles

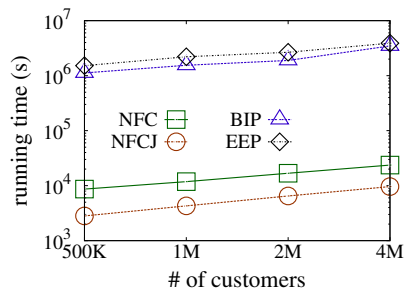

(c) Running time, Gaussian

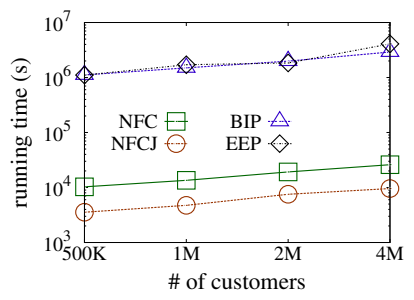

(f) Running time, Zipfian

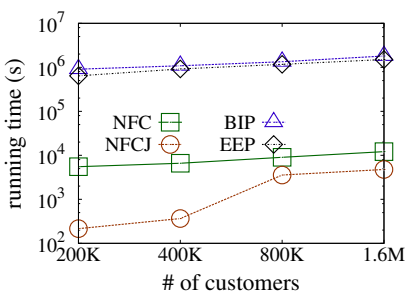

(i) Running time, Melbourne

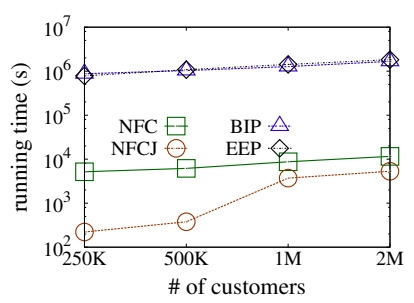

(1) Running time, Los Angeles

Fig. 18: Effect of the cardinality of the customer set $M$

level; it does not matter much if many nodes are overlapped with each other as grouping effect may diminish the disadvantage brought by overlapping nodes, e.g., extensive traversals will be shared. Overall, NFCJ and NFC beat SS, EEP, and BIP in orders of magnitude. NFC outperforms NFCJ in terms of CPU time when facility set grows bigger, NFCJ is still the best among all algorithm which achieve the lowest number of I/O operations and therefore the smallest running time cost in all cases.

Effect of customer cardinality $|M|$. More customers may increase the influence values of facilities and it is expected to consume more time as well as 
I/O operations. The results of varying the cardinality of $M$ are given in Fig. 18 . In all cases, the performance of all algorithms degrades as the cardinality of $M$ grows. The ranking of algorithms in terms of their efficiency still stays the same, i.e., NFCJ and NFC beat other algorithms by up to two orders of magnitude while BIP outperforms SS and SS outperforms EEP. It can also be observed that BIP is more steady when compared to SS when the cardinality of the customer set grows. The reason is that BIP tends to avoid querying the customer R-tree and does not extensively traverse this customer R-tree when pruning. Hence, the growth of customer does not directly affect the performance of BIP. To summarize, the results here are similar to what has been shown in previous experiments and fit to our theoretic analysis in previous sections.

\section{Conclusion}

We formulated a practical location selection problem using reverse nearest neighbor semantics and proposed a new type of location selection query named top- $k$ most influential location selection query. We attempted the popular approach, branch-and-bound, to process the query, namely Estimation Expanding Pruning (EEP) and Bounding Influence Pruning (BIP). As observed from both the evaluation, in terms of CPU time, BIP outperforms the sequential scan by several times while EEP is constantly outperformed by the sequential scan. Moreover, from analysis and evaluation, branch-and-bound algorithms achieve neither competitive worst-case complexity to the sequential scan nor good I/O performance. We proposed a new Nearest Facility Circle Join (NFCJ) algorithm, which serves as the best solution for the query based on the analysis of complexity for all algorithms. We further provided an extensive experimental study on all algorithms whose results confirm with the theoretic analysis. We draw the conclusion that, for the top- $k$ most influential location selection query, NFCJ is the most efficient algorithm in terms of both time and the number of I/O operations, out performing other solutions by orders of magnitude.

Acknowledgements This work is supported in part by the National Natural Science Foundation of China (No. 61272065), and the Natural Science Foundation of Guangdong Province, China (No. S2012010009311), the Fundamental Research Funds for the Central Universities, SCUT(Grant No. 2012ZZ0088), and the Australian Research Council (ARC) Discovery Project DP130104587. Dr. Rui Zhang is supported by the ARC Future Fellowships Project FT120100832. Zeyi Wen is supported by the Commonwealth Scientific and Industrial Research Organisation (CSIRO).

\section{References}

1. Achtert, E., Kriegel, H.P., Krger, P., Renz, M., Zfle, A.: Reverse k-nearest neighbor search in dynamic and general metric databases. In: Proc. of EDBT (2009)

2. ANNLibrary: http://www.cs.umd.edu/ mount/ann/ (2011) 
3. Aronovich, L., Spiegler, I.: Bulk Construction of Dynamic Clustered Metric Trees. Knowledge and Information Systems 22(2), 211-244 (2009)

4. Brinkhoff, T., Kriegel, H.P., Seeger, B.: Efficient processing of spatial joins using r-trees. In: Proc. of SIGMOD (1993)

5. Cabello, S., D, J.M., Langerman, S., Seara, C.: Reverse Facility Location Problems. In: Proc. of CCCG (2006)

6. Cheema, M.A., Lin, X., Wang, W., Zhang, W., Pei, J.: Probabilistic Reverse Nearest Neighbor Queries on Uncertain Data. IEEE TKDE 22(4), 550-564 (2010)

7. Cheema, M.A., Lin, X., Zhang, W., Zhang, Y.: Influence Zone : Efficiently Processing Reverse k Nearest Neighbors Queries. In: Proc. of ICDE (2011)

8. Cheema, M.A., Zhang, W., Lin, X., Zhang, Y.: Efficiently Processing Snapshot and Continuous Reverse k Nearest Neighbors Queries. The VLDB Journal (2012)

9. Chen, H., Liu, J., Furuse, K., Yu, J.X., Ohbo, N.: Indexing Expensive Functions for Efficient Multi-Dimensional Similarity Search. Knowledge and Information Systems 27(2), 165-192 (2010)

10. CloudMade: http://downloads.cloudmade.com/ (2013)

11. Du, Y., Zhang, D., Xia, T.: The Optimal-Location Query. Advances in Spatial and Temporal Databases 3633, 163-180 (2005)

12. Gao, Y., Zheng, B., Chen, G., Li, Q.: Optimal-Location-Selection Query Processing in Spatial Databases. IEEE TKDE 68(8), 1162-1177 (2009)

13. Ghaemi, P., Shahabi, K., Wilson, J.P., Banaei-Kashani, F.: Optimal network location queries. In: Proc. of GIS (2010)

14. Guttman, A.: R-Trees: A Dynamic Index Structure for Spatial Searching. In: Proc. of SIGMOD, pp. $47-57$ (1984)

15. Huang, J., Wen, Z., Qi, J., Zhang, R., Chen, J., He, Z.: Top-k Most Influential Location Selection. In: Proc. of CIKM (2011)

16. Korn, F., Muthukrishnan, S.: Influence Sets Based on Reverse Nearest Neighbor Queries. In: Proc. of SIGMOD (2000)

17. Mouratidis, K., Papadias, D., Papadimitriou, S.: Medoid Queries in Large Spatial Databases. In: Proc. of SSTD, pp. 55-72 (2005)

18. OpenStreetMap: http://www.openstreetmap.org/ (2013)

19. Qi, J., Zhang, R., Kulik, L., Lin, D., Xue, Y.: The Min-dist Location Selection Query. In: Proc. of ICDE (2012)

20. Roussopoulos, N., Kelley, S., Vincent, F.: Nearest Neighbor Queries. In: Proc. of SIGMOD, pp. 71-79 (1995)

21. Shang, S., Yuan, B., Deng, K., Xie, K., Zhou, X.: Finding the Most Accessible Locations - Reverse Path Nearest Neighbor Query in Road Networks Categories and Subject Descriptors. In: Proc. of GIS (2011)

22. SouFang: http://www.soufun.com (2013)

23. Stanoi, I., Riedewald, M., Agrawal, D., Abbadi, A.E.: Discovery of Influence Sets in Frequently Updated Database. In: Proc. of VLDB (2001)

24. Sun, Y., Huang, J., Chen, Y., Zhang, R., Du, X.: Location selection for utility maximization with capacity constraints. In: Proc. of CIKM (2012)

25. Tao, Y., Lian, X.: Reverse kNN Search in Arbitrary Dimensionality. In: Proc. of VLDB (2004)

26. trulia: http://trulia.com (2013)

27. Vaidya, P.M.: AnO(n logn) Algorithm for the All-Nearest-Neighbors Problem. Discrete and Computational Geometry 4(1) (1989)

28. Wong, R.C.W., Özsu, M.T., Fu, A.W.C., Yu, P.S., Liu, L., Liu, Y.: Maximizing bichromatic reverse nearest neighbor for $\mathrm{L} p$-norm in two- and three-dimensional spaces. The VLDB Journal 20(6), 893-919 (2011)

29. Wong, R.C.w., Ozsu, M.T., Yu, P.S., Fu, A.W.c., Liu, L.: Efficient Method for Maximizing Bichromatic Reverse Nearest Neighbor. In: Proc. of VLDB (2009)

30. Wu, W., Yang, F., Chan, C.Y., Tan, K.L.: FINCH : Evaluating Reverse k-NearestNeighbor Queries on Location Data. In: Proc. of VLDB (2008)

31. Xia, T., Zhang, D., Kanoulas, E., Du, Y.: On Computing Top-t Most Influential Spatial Sites. In: Proc. of VLDB (2005)

32. Yan, D., Wong, R.C.w., Ng, W.: Efficient Methods for Finding Influential Locations with Adaptive Grids. In: Proc. of CIKM, pp. 1475-1484 (2011) 
33. Yang, C., Lin, K.i.: An index structure for efficient reverse nearest neighbor queries. In: Proc. of ICDE, pp. 485-492 (2001)

34. Zhang, D., Du, Y., Xia, T., Tao, Y.: Progressive Computation of the Min-Dist Optimal Location Query. In: Proc. of VLDB (2006)

35. Zhang, J., Mamoulis, N., Papadias, D., Tao, Y.: All-Nearest-Neighbors Queries in Spatial Databases. In: Proc. of SSDM, pp. 297-306 (2004)

36. Zheng, K., Huang, Z., Zhou, A., Zhou, X.: Discovering the Most Influential Sites over Uncertain Data : A Rank Based Approach. IEEE TKDE (2011)

\section{Author Biographies}

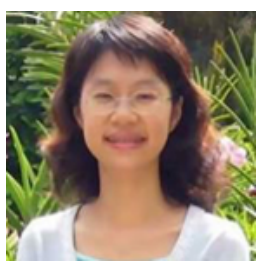

Jian Chen is currently an Associate Professor of the School of Software Engineering at South China University of Technology where she started as an Assistant Professor in 2005. She received her B.S. and Ph.D. degrees, both in Computer Science, from Sun Yat-Sen University, China, in 2000 and 2005 respectively. Her research interests can be summarized as developing effective and efficient data analysis techniques for complex data and the related applications. Particularly, she is currently interested in various techniques of data mining, Web search, information retrieval, recommendation techniques as well as their applications in social networks, business and bioinformatics. Since 2000, she has published 40 research papers in refereed journals and conferences, has served in the organization committees and the program committees of over 20 international conferences and workshops, and has been a reviewer for the leading academic journals in her fields. She has served as a PC member of SDM2013, PAKDD20092013, DASFFA2013, CIKM2009-2013, ADMA2009-2013, etc.

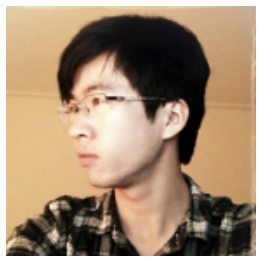

Jin Huang obtained his bachelor's degree in Software Engineering from South China University of Technology in 2011. Before commencing the research program under the supervision of Associate Professor Rui Zhang in Melbourne in 2012, He spent several months at GSegment Space Technologies in Beijing, China, working on projects led by National Astronomical Observatories and European Space Agency. His research interests include large-scale data analysis, spatio-temporal data mining, data structure and index techniques.

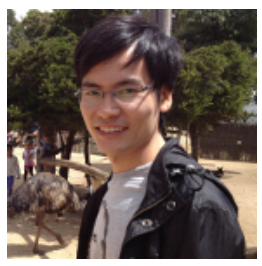

Zeyi Wen is a PhD Candidate at University of Melbourne, under the supervision of Associate Professor Rui Zhang and Dr. Kerry Taylor. He holds a Bachelor of Software Engineering from South China University of Technology in 2010. His areas of research include web data mining, high performance computing, and spatio-temporal data management. 

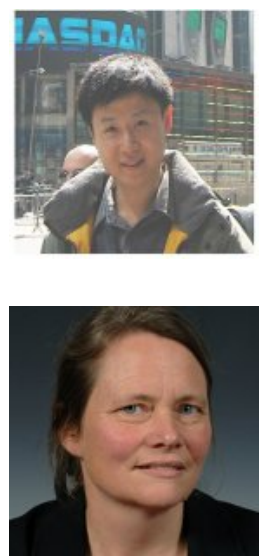

Zhen He is a senior lecturer in the Department of Computer Science at La Trobe University Australia. He obtained his undergraduate and $\mathrm{PhD}$ degrees in computer science from the Australian National University. His research interests include moving object databases, flash memory databases, relational database optimization and parallel databases.

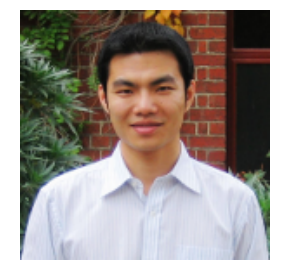

Rui Zhang is an Associate Professor and Reader in the Department of Computing and Information Systems at the University of Melbourne. He obtained his bachelor's degree from Tsinghua University in 2001 and his PhD from National University of Singapore in 2006. Before joining the University of Melbourne, he has been a visiting research scientist at AT\&T labs-research in New Jersey and at Microsoft Research in Redmond, Washington. Since January 2007, he has been a faculty member in the Department of Computing and Information Systems at the University of Melbourne. Recently, he has been a visiting researcher at Microsoft Research Asia in Beijing regularly collaborating on his ARC Future Fellowship project. His research interest is data and information management in general, particularly in areas of indexing techniques, moving object management, web services, data streams and sequence databases. 


\section{University Library}

\section{- M M N E R VA A gateway to Melbourne's research publications}

Minerva Access is the Institutional Repository of The University of Melbourne

Author/s:

Chen, J;Huang, J;Wen, Z;He, Z;Taylor, K;Zhang, R

Title:

Analysis and evaluation of the top-k most influential location selection query

Date:

2015-04-01

Citation:

Chen, J., Huang, J., Wen, Z., He, Z., Taylor, K. \& Zhang, R. (2015). Analysis and evaluation of the top-k most influential location selection query. KNOWLEDGE AND INFORMATION SYSTEMS, 43 (1), pp.181-217. https://doi.org/10.1007/s10115-013-0720-0.

Persistent Link:

http://hdl.handle.net/11343/54736 